\title{
Global fitting of globular cluster age indicators ${ }^{\star}$
}

\author{
F. Meissner ${ }^{1}$ and A. Weiss ${ }^{1}$ \\ Max-Planck-Institut für Astrophysik, Karl-Schwarzschild-Str. 1, 85748 Garching, Germany
}

e-mail: [meissner;weiss] @mpa-garching.mpg.de

Received 3 March 2006 / Accepted 12 June 2006

\section{ABSTRACT}

\begin{abstract}
Context. Stellar models and the methods for the age determinations of globular clusters are still in need of improvement.
Aims. We attempt to obtain a more objective method of age determination based on cluster diagrams, avoiding the introduction of biases due to the preference of one single age indicator.

Methods. We compute new stellar evolutionary tracks and derive the dependence of age indicating points along the tracks and isochrone - such as the turn-off or bump location - as a function of age and metallicity. The same critical points are identified in the colour-magnitude diagrams of globular clusters from a homogeneous database. Several age indicators are then fitted simultaneously, and the overall best-fitting isochrone is selected to determine the cluster age. We also determine the goodness-of-fit for different sets of indicators to estimate the confidence level of our results.

Results. We find that our isochrones provide no acceptable fit for all age indicators. In particular, the location of the bump and the brightness of the tip of the red giant branch are problematic. On the other hand, the turn-off region is very well reproduced, and restricting the method to indicators depending on it results in trustworthy ages. Using an alternative set of isochrones improves the situation, but neither leads to an acceptable global fit.

Conclusions. We conclude that evolutionary tracks of low-mass metal-poor stars are far from reproducing all aspects of globular cluster colour-magnitude diagrams and that the determination of cluster ages still depends on the favourite method or indicator chosen.
\end{abstract}

Key words. stars: evolution - Galaxy: globular clusters: general

\section{Introduction}

The determination of globular cluster (GC) ages rests on the fact that colour-magnitude diagrams (CMDs) of single-age singlecomposition stellar populations exhibit specific time-dependent features. Most importantly, this is the location of the turn-off (TO), which - together with the cluster's distance - serves as the most straightforward and widely used age indicator. However, there are other parts of the CMD that change their colour or brightness with age, too. Since the sensitivity to time is different for the various parts of the cluster CMD, it is possible to use either the various indicators independently, or the differences in colour and brightness between pairs of them; these latter methods have the advantage of being independent of distance. Finally, one may attempt to fit, within constraints, several properties of parts of the cluster diagram with one isochrone, which is the method of VandenBerg (2000) or even fit the whole fiducial cluster line, as done by Straniero \& Chieffi (1991).

The decision of which age indicator is used is the choice of the investigator and may depend on the observational data (e.g., CMDs not deep enough prevent the use of the TO), the isochrones (with or without the horizontal branch), or simply tradition. In the epoch of rather accurate Hipparcos distances, the traditional TO brightness has experienced new interest (Gratton et al. 1997; Carretta et al. 2000). Salaris \& Weiss $(1997,2002)$ prefer the brightness difference between horizontal branch (HB) and TO for absolute ages, and the colour difference between $\mathrm{TO}$ and red giant branch (RGB) for relative ages, while

* Tables 6 to 9 are only available in electronic form at http://www.edpsciences.org
Rosenberg et al. (1999) use the former method for an almost empirical relative age scale. Rarely, several age indicators are used simultaneously to check for consistency. Therefore, the choice of the age determination method is subject to a bias.

The aim of this paper is to investigate whether the simultaneous use of several age indicators, which implies a higher degree of objectivity, is possible and whether it leads to overall acceptable isochrone fits. We restrict ourselves to brightness and colour differences, thereby avoiding the problem of distance accuracy. We do not intend to present new or improved age determinations, but rather to concentrate on developing a new and more reliable method. The basic idea is to define a selection of crucial points along the cluster sequences and the corresponding ones along theoretical isochrones. These are the TO, and two points close to but cooler than it, the bump and tip of the RGB, and the colour of the lower RGB. HB and RGB tip are almost age independent and serve as reference points; they also provide a means of predicting the distance to the cluster. This can be compared to independent distance measurements to check for consistency and for graphical overlay of isochrone and cluster ridge line.

Various brightness and colour differences are defined according to their use in the literature as age indicators. They are derived directly from the photometric data; theoretical values are determined from our isochrones, which we computed specifically for this project, and their age dependence fitted by simple analytical formulae. The deviation between theoretical and observed value as a function of age enters a goodness-of-fit test (a $\chi^{2}$-method). The overall minimum of this function determines the best age, and its value the confidence level. The basic result is that using all indicators results in flat minima with low 


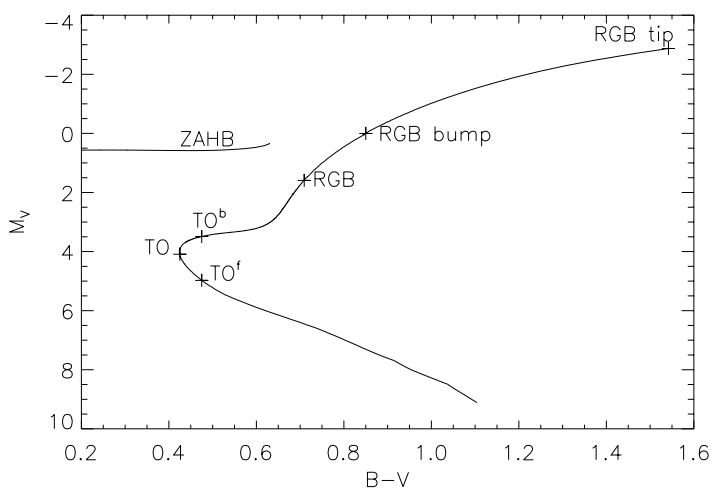

Fig. 1. A sample isochrone $\left(\log Z / Z_{\odot}=-3 ; t=12 \mathrm{Gyr}\right)$ in the observable plane and points along it used for the age indicators.

probability, while the restriction to subsets of indicators (equivalent to putting zero weight to the others) allows quite precise age determinations. The reason for the failure of the complete set of age indicators lies in the inability of the isochrones to fit not just the overall shape, but the individual features of a cluster CMD. More details about our work can be found in Meissner (2005) on which this paper is based.

The age indicators will be introduced and discussed in Sect. 2 with references to earlier work using them. The observational data are presented in Sect. 3 along with the details of the determination of the ridge line and the various features of interest. Then (Sect. 4) follows a short description of our calculations and a set of stellar tracks. Section 5 contains the presentation of results, while the discussion in Sect. 6 closes the paper.

\section{Age indicators from cluster CMD}

\subsection{Indicator points}

Turn-off region: The classical age indicator (Renzini \& Fusi Pecci 1988) in cluster CMDs is the turn-off (indicated in Fig. 1 by “TO"), whose position moves to the lower right (cooler and fainter) with age. Its colour can be determined easily and accurately, if the reddening is small or well-determined, while the brightness is more difficult to fix due to the long and almost vertical extension of the ridge-line (or, equivalently, the isochrone), which can reach up to $0.1 \mathrm{mag}$, corresponding to an age uncertainty of about 1 Gyr. In this paper, we will always indicate TO quantities by the corresponding index.

To avoid the problem of determining $V_{\mathrm{TO}}$ accurately, two alternative indicator points have been introduced that are 0.05 mag redder than the TO, but that lie on less steep portions of the isochrone: the point $\mathrm{TO}^{\mathrm{b}}$ on the post-TO part and $\mathrm{TO}^{\mathrm{f}}$ on the main sequence. The first alternative was introduced by Chaboyer et al. (1996b); it has the same age sensitivity as the TO itself, but its determination is claimed to be a factor of 2 better. The second one was proposed by Buonanno et al. (1998) and in addition is claimed to be less affected by uncertainties such as the convection theory. While this conjecture is plausible on the basis that pre-TO stars are less evolved, it was never investigated in detail.

Red giant branch: The luminosity of the red giant branch tip and its brightness in the $I$-band, $M_{I \text {,tip }}$, depend only weakly on composition, and hardly at all on age. Therefore, it is used as a standard candle to determine distances to galaxies with resolved populations (see Salaris \& Cassisi 1997, 1998 for a discussion). Since the observational data we are using (Piotto et al. 2002 b, Sect. 3) provide only $B$ and $V$ magnitudes, we will

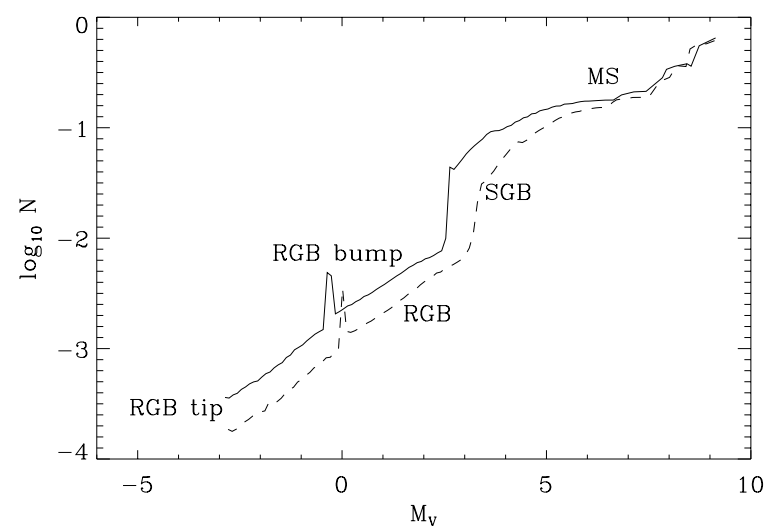

Fig. 2. Luminosity function isochrones for $\log Z / Z_{\odot}=-3$ and ages of 6 (solid line) and $12 \mathrm{Gyr}$ (dashed). Along the LF main-sequence (MS), the sub-giant branch (SGB), red giant branch (RGB), tip of the RGB, and position of the RGB bump are indicated.

employ the more metallicity-dependent $V_{\text {tip }}$ as the reference point from which brightness differences to time-dependent points can be defined.

On the lower red-giant branch, the colour of a point or region is used for colour-based age indicators (also called "horizontal" indicators, in contrast to the brightness-based "vertical" ones). We chose the colour of a point 2.5 mag brighter than the TO $\left((B-V)_{\mathrm{RGB}}\right)$; Salaris \& Weiss (1997) used the mean colour for a range of about $0.5-1.0 \mathrm{mag}$ starting from this point. The uncertainty of $V_{\mathrm{TO}}$ is not very crucial since the RGB colour hardly changes over $0.1 \mathrm{mag}$.

The most age-sensitive feature along the RGB is the socalled bump, where the evolutionary sequence backtracks on itself over a small magnitude range when the H-burning shell reaches the layers previously mixed by convection at its deepest penetration. This results observationally in an enhanced number of stars in a limited magnitude range (see Salaris et al. 2002 for more details). The bump is now routinely found in high-quality cluster photometry, both in the optical (Riello et al. 2003) and near infrared (Valenti et al. 2004). Its location both in colour and brightness is mass-, and therefore age-dependent. It also depends on composition.

As the bump has to be detected from the luminosity function (LF) of a cluster, we show the simulated LFs of isochrones with $\log Z / Z_{\odot}=-3$ for ages of 6 and 12 Gyr in Fig. 2. For the initial mass function (IMF) needed to produce the LF, the standard Salpeter law (Salpeter 1955) was assumed.

Horizontal branch: The brightness of the zero-age horizontal branch (ZAHB) is only very weakly dependent on age, and therefore $V_{\text {ZAHB }}$ is widely used for vertical age indicators (VandenBerg et al. 1990; Salaris \& Weiss 1997). Theoretically it is easily determined, but observationally one estimates it from the lower envelope of the horizontal branch. From $V_{\mathrm{ZAHB}}$, one can thus derive the distance to the cluster; in general, the agreement with main-sequence fitting distances is quite good (Salaris $\&$ Weiss 1998). The colour of the HB red end is another possible age-dependent feature, but as it is sensitive to many factors, and due to our lack of understanding HB morphology, we chose not to use it.

\subsection{Age indicators}

$\Delta V_{\mathrm{TO}}^{\mathrm{ZAHB}}$ : the difference between $\mathrm{TO}$ and ZAHB, $\Delta V_{\mathrm{TO}}^{\mathrm{ZAHB}}=$ $V_{\mathrm{TO}}-V_{\mathrm{ZAHB}}$, is the most widely used vertical age indicator (see 


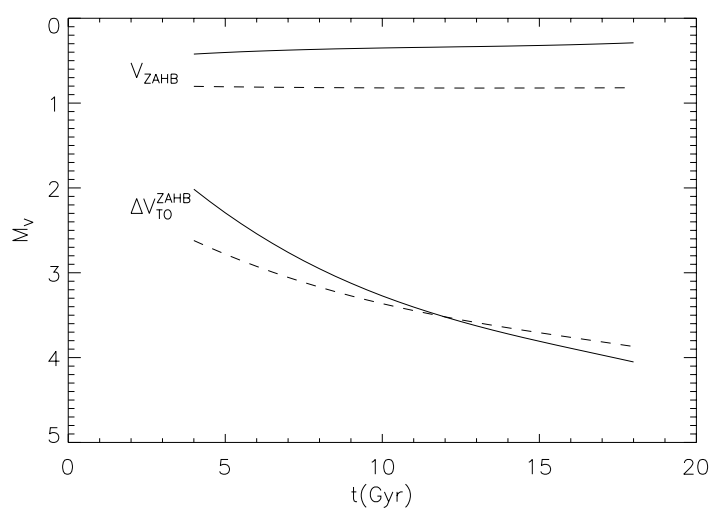

Fig. 3. $\Delta V_{\mathrm{TO}}^{\mathrm{ZAHB}}$ (upper lines) and $V_{\mathrm{ZAHB}}$ (lower lines) for $Z=2 \times$ $10^{-4}$ (solid) and $10^{-2}$ (dashed) as function of time from our theoretical isochrones.

De Angeli et al. 2005, for the most recent application), and probably the most reliable one. If $V_{\mathrm{ZAHB}}$ is taken at the same colour as the TO, it is largely independent of uncertainties in the colour transformations or reddening, and depends only on the relative accuracy of bolometric corrections, as was discussed by Salaris $\&$ Weiss (1998). In contrast to that paper and to Salaris \& Weiss (2002), we also use $\Delta V_{\mathrm{TO}}^{\mathrm{ZAH}}$ for those clusters, which do not have stars on the HB at the TO colour. This requires a less accurate approach: we chose to select the ZAHB level at the closest colour available in the observed CMD. As long as this point is on the horizontal part of the ZAHB, this appears to be rather safe, but for an extremely blue morphology, it would be less so. Figure 3 shows $\Delta V_{\mathrm{TO}}^{\mathrm{ZAHB}}$ as a function of age, as well as $V_{\mathrm{ZAHB}}$ for two metallicities, as obtained from the theoretical isochrones. It is obvious that $V_{\mathrm{ZAHB}}$ is nearly constant over an age range of 8-15 Gyr.

$\Delta V_{\mathrm{TOb}}^{\mathrm{ZAHB}}$ and $\Delta V_{\mathrm{TOf}}^{\mathrm{ZAHB}}$ : these are the two alternative age indicators equivalent to $\Delta V_{\mathrm{TO}}^{\mathrm{ZAHB}}$, but without the uncertainty connected with the determination of $V_{\mathrm{TO}}$. All other comments made in the previous paragraph hold. The age and metallicity dependence is very similar to that of $\Delta V_{\mathrm{TO}}^{\mathrm{ZAHB}}$.

$\Delta V_{\mathrm{TO}}^{\mathrm{Bump}}$ : in case of a $\mathrm{HB}$ with a morphology rendering the previous age indicators inaccurate, one can resort to $\Delta V_{\mathrm{TO}}^{\mathrm{Bump}}=$ $V_{\text {TO }}-V_{\text {Bump }}$ instead. Its age sensitivity is slightly lower than that of $\Delta V_{\mathrm{TO}}^{\mathrm{ZAHB}}$ for low ages, but almost comparable at the interesting age range around $10 \mathrm{Gyr}$. The problems with this indicator lie in the need for photometry of a sufficient number of stars to identify the bump. Theoretically, as we will see, it appears to be systematically too bright by a few tenths of a magnitude as is also found in some observational papers (e.g., Zoccali \& Piotto 2000).

$\Delta V_{\mathrm{TO}}^{\mathrm{tip}}: \Delta V_{\mathrm{TO}}^{\mathrm{tip}}=V_{\mathrm{TO}}-V_{\text {tip }}$ is again useful in cases of an insufficiently populated or observed HB. It is particularly sensitive to age for lower metallicities, changing by more than 0.5 mag between 8 and 14 Gyr. It was used by Vandenberg \& Durrell (1990) for relative age determinations at a given $[\mathrm{Fe} / \mathrm{H}]$. The obvious difficulty lies in a precision estimate of $V_{\text {tip }}$, since the number of stars at the tip is very low, due to the increased evolutionary speed in this phase.

$\Delta V_{\mathrm{ZAHB}}^{\mathrm{Bump}}$ : if the photometry is not deep enough to reach the $\mathrm{TO}$, it is in principle possible to use $\Delta V_{\mathrm{ZAHB}}^{\mathrm{Bump}}=V_{\mathrm{Bump}}-V_{\mathrm{ZAHB}}$ (Ferraro et al. 1999). The age sensitivity is very weak, amounting to $\approx 0.5 \mathrm{mag}$ over $10 \mathrm{Gyr}$ only, such that $\Delta V_{\mathrm{ZAHB}}^{\mathrm{Bump}}$ will not provide any additional age restriction in comparison with the other

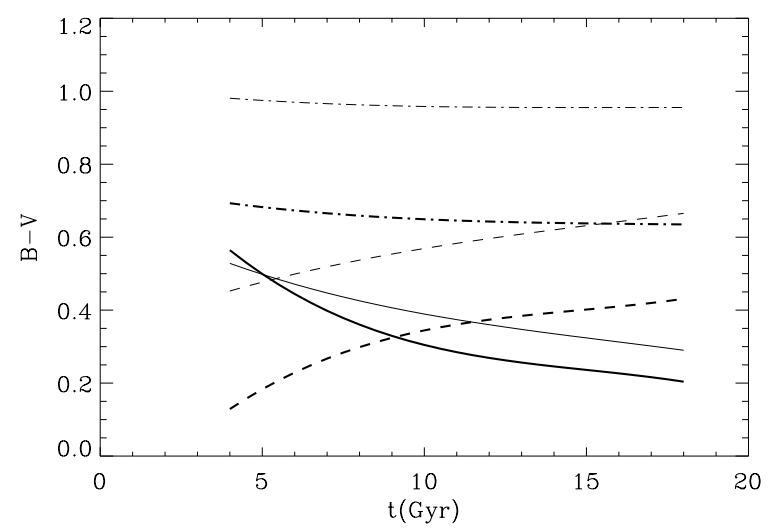

Fig. 4. $\Delta(B-V)_{\mathrm{TO}}^{\mathrm{RGB}}$ (solid lines), $(B-V)_{\mathrm{TO}}$ (dashed), and $(B-$ $V)_{\mathrm{RGB}}$ (dash-dotted) from our isochrones. All quantities are given for two metallicities, $Z=2 \times 10^{-4}$ (thick lines) and $10^{-2}$ (thin lines).

indicators (Cassisi \& Salaris 1997). The colour at which $V_{\mathrm{ZAHB}}$ is to be taken is arbitrary, but must be consistent between data and isochrone.

$\Delta(B-V)_{\mathrm{TO}}^{\mathrm{RGB}}$ : the preferred horizontal (here in $\left.(B-V)\right)$ age indicator is $\Delta(B-V)_{\mathrm{TO}}^{\mathrm{RGB}}=(B-V)_{\mathrm{RGB}}-(B-V)_{\mathrm{TO}}($ VandenBerg et al. 1990; Salaris \& Weiss 1997; Buonanno et al. 1998). As mentioned in Sect. 2.1, we take $(B-V)_{\mathrm{RGB}}$ at a brightness 2.5 mag brighter than the TO. This point represents a compromise between minimising photometric errors and stellar density on the RGB (Rosenberg et al. 1999). The general problems with colours rest with the uncertainties in both the calculations (convection theory) and the transformations between theoretical $T_{\mathrm{eff}}$ and colour. However, by using a differential quantity, systematic uncertainties related to the models' metallicity can probably be avoided. A further reduction of the influence of these uncertainties can be achieved by using $\Delta(B-V)_{\mathrm{TO}}^{\mathrm{RGB}}$ for relative ages, as in Salaris \& Weiss (1998).

$\Delta(B-V)_{\text {TO }}^{\text {Bump }}$ : alternatively, the colour difference between bump and TO can be used, which is very similar to $\Delta(B-V)_{\mathrm{TO}}^{\mathrm{RGB}}$, but is taken at the location of a physical feature.

Hatzidimitriou (1991) presented a further "age calibrator", using the colour difference between RGB and the red part of the HB. However, in view of the variety of HBs we encounter in a sample of GC and the unknown influence of the second parameter, we chose not to include this in our investigation.

\section{Cluster data}

\subsection{Cluster sample}

This work is concerned with developing a more complete approach to globular cluster dating. The observational data should be comprehensive in terms of metallicity range and consistent to avoid differential effects in the quality of the method, which could be due to the differences in the database. For this reason, the photometric cluster catalogues by Rosenberg et al. (2000b) or Piotto et al. (2002a) are excellent choices. The first catalogue was used by Salaris \& Weiss (2002), and we decided to use the second one, which contains 74 galactic GCs in the HST F439W and F555W filters. The HST observations concentrated in particular on the central parts of the clusters. We obtained the data from the Padua web page ${ }^{1}$. The catalogue conveniently offers reddening-corrected Johnson colours $(B, V)$,

\footnotetext{
${ }^{1}$ http://dipastro.pd.astro.it/globulars
} 


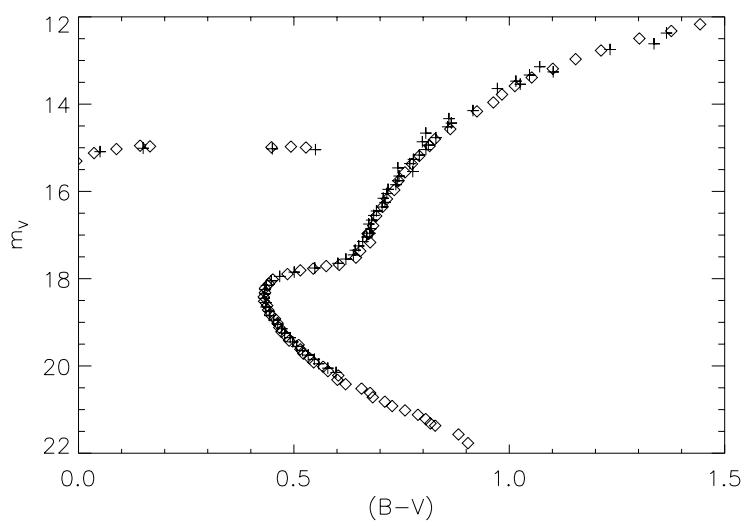

Fig. 5. Mean ridge line for the cluster M 5 as determined by us (crosses) and by Sandquist et al. (1996) from their own data (diamond symbols).

which were obtained by Piotto et al. (2002a) from an iterative data calibration. While the authors do not claim the reddening to be always correct, in particular in the case of highly reddened clusters, our differential age indicators are mostly rather insensitive to reddening.

We did not select 28 clusters due to the low number of stars, a significant scatter in the CMDs, or a lack of any discernible HB. The final number of clusters used was therefore 46 (Table 6).

For the metallicities $([\mathrm{Fe} / \mathrm{H}])$, we used the values on the scale of Zinn \& West $\left(1984,[\mathrm{Fe} / \mathrm{H}]_{\mathrm{ZW}}\right)$ given in the cluster catalogue, and transformed them to the Carretta \& Gratton (1997, $[\mathrm{Fe} / \mathrm{H}]_{\mathrm{CG}}$ ) scale with the relation given by Carretta et al. (2001), unless they were available from Rutledge et al. (1997).

\subsection{Data extraction}

\subsubsection{Mean ridge line}

The first step in preparing the photometric data for our method is to determine the mean ridge line. After the exclusion of obvious HB stars by eye, we follow the standard procedure described, e.g., in Cho et al. (2005): After determining the mean colour of stars in brightness bins of $0.25 \mathrm{mag}$, stars with a colour more than $2.5 \sigma$ different from the mean are removed. Then, the determination of mean and variance are repeated until convergence is reached, usually in less than 5 iterations. Typically, around 5\% of the initial non-HB objects are removed. We also tested data using the median instead of the mean, but the difference in the final ridge line was not recognizable.

Figure 5 shows, as an example, our ridge line and that of Sandquist et al. (1996) for the cluster M 5. It also shows that along the RGB, the ridge line data points do not form a smooth line due to statistical variations at low star numbers per brightness interval. Therefore, we performed a hyperbolic fit to the data following Rosenberg et al. (1999) and Saviane et al. (2000).

\subsubsection{ZAHB brightness}

The ZAHB brigthness was determined using the method presented by Salaris \& Weiss (1997): the HB is divided into colour bins with width sufficiently large to contain at least 10 stars. Each colour bin is then sub-divided into brightness bins of $0.05 \mathrm{mag}$, and the number of stars in each of them is used for a histogram of the colour bin. The criterion for the ZAHB is that $90 \%$ or more of all stars are above the ZAHB and that the

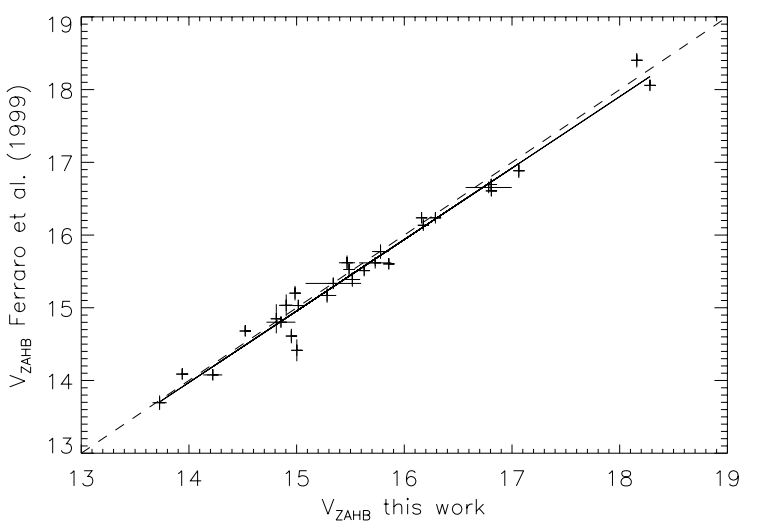

Fig. 6. Comparison of our derived values for $V_{\mathrm{ZAHB}}$ and those obtained by Ferraro et al. (1999) for clusters in common. The solid line is a linear fit through the data points, and the dashed one the 1:1 line for comparison.

number of stars drops by at least a factor of two from the bin above to that below the ZAHB level. The error is estimated from the brightness width. Not all colour bins allow the determination of the ZAHB at this colour and under these conditions, and thus the ZAHB line is obtained by interpolation between those colours where the method is successful. To check the reliability of our method, we compared our results with those of Ferraro et al. (1999), who determined, among other parameters, $V_{\mathrm{ZAHB}}$ for 61 GCs. Figure 6 shows the result of the comparison, which confirms our method completely.

$V_{\text {ZAHB }}$ can be compared directly with the theoretical predictions (Sect. 4.2), and therefore immediately yields a distance to the cluster. We have also compared these distances to those by Ferraro et al. (1999) and found that our distance moduli are on average $0.1 \mathrm{mag}$ larger. This is the immediate consequence of the Straniero et al. (1997) stellar models used by Ferraro et al. (1999). We emphasize again that the distances are not necessary for any age indicator we are using, but are just for the purpose of comparing isochrone with data in the CMD.

\subsubsection{The turn-off region}

For the cluster TO, one should select the bluest point along the MS. However, as disussed by Salaris et al. (1997) for the case of M 15, the uncertainties in the mean colour of brightness bins, together with the near-vertical CMD, introduces an uncertainty of 0.1 to $0.2 \mathrm{mag}$. We followed the method by Chaboyer et al. (1996a), who fitted the mean ridge line around the TO by a fifthorder polynomial $(B-V)=f(V)$, and then took the bluest point along this curve as the TO. This point does not necessarily have the same colour as the bluest point of the ridge line. The error $\sigma(B-V)_{\mathrm{TO}}$ introduced is estimated (Press et al. 1992) by the expression

$\sigma(B-V)_{\mathrm{TO}}=\sqrt{\frac{1}{N} \sum_{i=1}^{N}\left(x_{i}-\tilde{x}_{i}\right)^{2}}$

Here, $N$ is the number of ridge points used for the fit, and $x_{i}$ and $\tilde{x}_{i}$ are the measured and the fitted colours in each bin. It is implied that this error is also the typical error of the TO colour, 


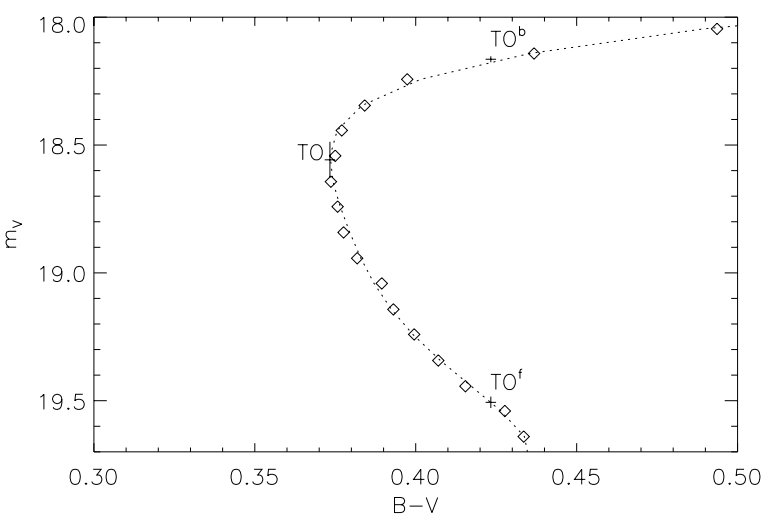

Fig. 7. Fit to the mean ridge line of NGC 362 in the TO region, and the location of $\mathrm{TO}, \mathrm{TO}^{\mathrm{b}}$, and $\mathrm{TO}^{\mathrm{f}}$ with their respective errors.

$\sigma(B-V)_{\mathrm{TO}}$, assuming that the polynomial fits the TO region well. The error in $V_{\mathrm{TO}}$ can then be estimated ${ }^{2}$ by

$\sigma\left(V_{\mathrm{TO}}\right)=\frac{\sigma_{\mathrm{fit}}}{\sqrt{N}}$

where $\sigma_{\text {fit }}$ is the standard deviation in brightness of the $N$ ridge points within a $3 \sigma(B-V)_{\mathrm{TO}}$ interval in $(B-V)$ around the TO. The formal errors are typically $0.1 \mathrm{mag}$ in $V_{\mathrm{TO}}$, and $0.001 \mathrm{mag}$ in $(B-V)_{\mathrm{TO}}$.

The related quantities $V_{\mathrm{TO}}^{\mathrm{b}}$ and $V_{\mathrm{TO}}^{\mathrm{f}}$ are both determined from the same polynomial fit and TO colour, with a similar estimate of the errors (see Fig. 7 for an illustrative example). Again, we compared our results with others from the literature; in this case we used Rosenberg et al. (1999), who determined $V_{\mathrm{TO}}$ from ground-based $V I$-photometry, for 16 clusters in common. Only in two cases, NGC 1261 and NGC 6093 (M 80), is there a difference of $0.3 \mathrm{mag}$. In all other cases, the agreement is of the order of $0.1 \mathrm{mag}$ or better; the rms deviation is 0.125 ( 0.073 without NGC 1261 and $\mathrm{M} 80)$. Noting that $\sigma\left(V_{\mathrm{TO}}\right)$ is $0.06-0.09$ for all these clusters, the agreement is excellent.

Finally, we compared our $\Delta V_{\mathrm{TO}}^{\mathrm{ZAH}}$ values with those by De Angeli et al. (2005), obtained from the same HST photometry. Most values agree within the error bars (the typical error in De Angeli et al. 2005 is around $0.08 \mathrm{mag}$ ), but there are some prominent outliers; many of them (like NGC 4147, NGC 6273, and NGC 6544) have very blue HBs, for which our method is more uncertain. For the 35 clusters, for which we have $\Delta V_{\mathrm{TO}}^{\mathrm{ZAHB}}$ from both sources, the rms is $0.15 \mathrm{mag}$. De Angeli et al. (2005) also obtained $\Delta V_{\mathrm{TO}}^{\mathrm{ZAHB}}$ for the ground-based data by Rosenberg et al. (1999); the comparison there resulted in an rms deviation of only $0.09 \mathrm{mag}$. This better agreement is most likely due to the use of the same method of "template HBs" for determining the HB levels.

\subsubsection{The red giant branch bump}

To identify the RGB bump, the luminosity function (omitting HB and AGB stars) is needed. Piotto et al. (2002a) investigated the completeness of the luminosity function (LF) by artifical star experiments, concluding that the lower MS is complete only to a level of 0.7. In principle, LFs can be used for age determinations, as they allow the identification of the subgiant-branch and

\footnotetext{
2 Basic statistical properties and relations can be found in many textbooks on the subject; throughout this paper we used the one by Brandt (1999).
}

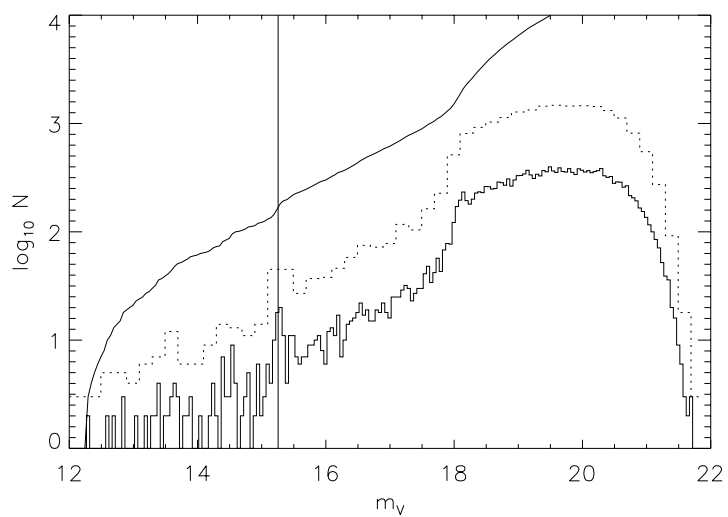

Fig. 8. Observed luminosity function (LF) for NGC 362. The lower lines are the differential LF with brightness bins of sizes $0.05 \mathrm{mag}$ (solid) and $0.2 \mathrm{mag}$ (dotted), and the upper solid line the integrated LF. The bump is indentified by the vertical line, where a slope change in the integrated function conincides with a local overdensity of stars.

bump luminosity and the relative number of stars in different luminosity bins, which is age-sensitive, too. Also, the shape of the LF at the subgiant-branch is age-dependent, and this was used by Zoccali \& Piotto (2000) to determine the ages of 18 clusters. However, incompleteness severely affects the usefulness of the $\mathrm{LF}$, and therefore we did not pursue this route any further.

To locate the bump, we used brightness bins of $0.05 \mathrm{mag}$, and followed Ferraro et al. (1999) and Riello et al. (2003) by requiring the coincidence of a stellar overdensity in a brightness bin and of a change of slope in the integrated LF. Figure 8 shows the example of NGC 362. For ten clusters in the sample, the bump could not be identified due to the large scattering of star numbers along the RGB (Table 7). The colour of the bump was determined by using the average $(B-V)$ of the bump and the two adjacent bins. The error was taken to be of the same size as the standard deviation of colours within these three bins. The theoretical counterpart was obtained by taking the upper bump brightness in the isochrone, which corresponds to the peak in the theoretical LF. Tables 6 and 7 list the brightness and colour of our age indicator points for all clusters taken into consideration.

\section{Stellar models and isochrones}

\subsection{Stellar evolution code}

For this project, we computed new stellar models and isochrones with the Garching stellar evolution code described by Weiss \& Schlattl (2000). The code incorporates the OPAL equation of state (Rogers et al. 1996) and the OPAL opacity tables (Iglesias \& Rogers 1996), supplemented by the molecular opacities of Alexander \& Ferguson (1994). In the calculations presented here, the equation of state (EOS) of Irwin (see Cassisi et al. 2003) has been used, which is based on the Eggleton et al. (1973) EOS, calibrated to the OPAL EOS. Diffusion of hydrogen and helium was taken into account using the diffusion coefficient treatment of Thoul et al. (1994), while that of heavier elements was ignored as it only has a minor effect on the evolution (see Weiss \& Schlattl 2000). Convection is treated according to standard mixing length theory with the Schwarzschild criterion for stability. The mixing length parameter was obtained from a calibration of the solar model (with diffusion), which yielded a value of 1.71. For the solar mixture, the values by Grevesse \& Noels (1993) were employed. No mass loss is included. 
Table 1. Chemical composition of stellar models with total metallicity $Z$, initial helium $Y_{0}$, hydrogen abundance $X_{0}$, iron abundance with respect to hydrogen (in spectroscopic notation $[\mathrm{Fe} / \mathrm{H}]$ ) and total metallicity with respect to hydrogen $[\mathrm{M} / \mathrm{H}]$.

\begin{tabular}{r|rrrr}
\hline \hline$Z$ & $Y_{0}$ & $X_{0}$ & {$[\mathrm{Fe} / \mathrm{H}]$} & {$[\mathrm{M} / \mathrm{H}]$} \\
\hline $2 \times 10^{-4}$ & 0.2455 & 0.7543 & -2.26 & -1.97 \\
$3 \times 10^{-4}$ & 0.2458 & 0.7539 & -2.08 & -1.79 \\
$6 \times 10^{-4}$ & 0.2465 & 0.7529 & -1.78 & -1.49 \\
$10^{-3}$ & 0.2475 & 0.7515 & -1.56 & -1.27 \\
$2 \times 10^{-3}$ & 0.2500 & 0.7480 & -1.25 & -0.96 \\
$3 \times 10^{-3}$ & 0.2525 & 0.7445 & -1.07 & -0.78 \\
$6 \times 10^{-3}$ & 0.2600 & 0.7340 & -0.77 & -0.48 \\
$10^{-2}$ & 0.2700 & 0.7200 & -0.54 & -0.25 \\
\hline
\end{tabular}

Nuclear reaction rates for $\mathrm{H}$-burning are mostly from Adelberger et al. (1998); the $\mathrm{S}$-factor for ${ }^{14} \mathrm{~N}(\mathrm{p}, \gamma){ }^{15} \mathrm{O}$ was taken from the recent result by the LUNA collaboration (Formicola et al. 2004), and that for ${ }^{3} \mathrm{He}\left({ }^{3} \mathrm{He}, 2 \mathrm{p}\right)^{4} \mathrm{He}$ from Junker et al. (1998). Helium burning reaction rates are by Caughlan et al. (1985) and Caughlan \& Fowler (1988).

\subsection{Models calculated}

We calculated models in the mass range of $M / M_{\odot}=$ $0.3, \ldots(0.1) \ldots 1.3$. For the initial helium content $Y_{0}$ we used

$Y_{0}=Y_{\mathrm{p}}+\frac{\Delta Y}{\Delta Z} Z$

with $Y_{\mathrm{p}}=0.245$ in agreement with the current concordance cosmological parameters and $\Delta Y / \Delta Z=2.5$. Enhancement of $\alpha$ elements is taken into account. Our choice of the enhancement factors was guided by Table 1 in Salaris \& Weiss (1998), and is similar, but slightly smaller than theirs. Most $\alpha$-elements are enhanced by $0.30-0.35$ dex, and oxygen by 0.40 dex. The particular choice is not significant at this low metallicity (Salaris \& Weiss 1998). At the fixed metallicity $Z, \alpha$-enhancement implies a reduced value for $[\mathrm{Fe} / \mathrm{H}]$ (see Weiss \& Schlattl 2000 for a table), as compared to solar-scaled mixtures. Total metallicities are given in Table 1 .

Our age indicators require theoretical ZAHB models, which we obtained by the method presented in Serenelli \& Weiss (2005). Briefly, for each metallicity, one stellar evolution model is followed completely from ZAMS to HB, including the full core helium flash, and without mass loss. From the ZAHB configuration of this model, other ZAHB models of lower mass are obtained by reducing the envelope mass. Serenelli \& Weiss (2005) have shown that this method reproduces models obtained from full calculations with high accuracy. As the ZAHB brightness hardly depends on age (a change in age of 6 Gyr results in a change in $\log L / L_{\odot}$ of only 0.02 ), it suffices to calculate one ZAHB per metallicity case for the age range of interest.

\subsection{Isochrones}

To obtain isochrones from the stellar evolution tracks, we follow the method of equivalence points (EP) described, e.g., in Bergbusch \& VandenBerg (1992). We defined 16 EPs in total, 5 on the MS, 7 more from the TO to the bottom of the RGB, and 4 more along the RGB, including 2 at the start and end of the bump. Between the EPs, additional points, their number ranging from 10 on the MS to 100 on the RGB, were distributed at equal stellar age increments (for details, see Meissner 2005). This procedure yields a "normalised track" of given mass and metallicity. To get an isochrone of age $t$ from these normalised tracks, $T_{\text {eff }}(t)$ and $\log L(t) / L_{\odot}$ values were interpolated for each available mass value. This results in a number of normalised points where the age of the lower mass track is higher and that of the higher mass track is lower than $t$. Additional isochrone points are thus obtained by interpolating $T_{\text {eff }}$ and $\log L / L_{\odot}$ in $t$ between corresponding normalised points. We constructed isochrones of age $t=4.0, \ldots(0.5) \ldots 18.0$ Gyr for each metallicity.

Finally, isochrones for the cluster metallicity are needed (measured by its $[\mathrm{Fe} / \mathrm{H}]$ value). Linear interpolation in metallicity was done between corresponding points on the available isochrones. We tested our method by interpolating and comparing with available metallicities, and found an accuracy of the order of $0.001 \mathrm{mag}$ in colour, resp. 0.01 in brightness.

As a further test of our models, we compared $T_{\text {eff }}$ of the isochrones with temperatures of globular cluster RGBs by Frogel et al. (1981). We find that with our standard mixing length parameter, our RGB temperatures are hotter than the emipircal ones for low metallicities $([\mathrm{Fe} / \mathrm{H}]=-2.1, \mathrm{M} 92)$ by about $100 \mathrm{~K}$. At $[\mathrm{Fe} / \mathrm{H}]=-1.3(\mathrm{M} 13$ and $\mathrm{M} 3)$, they match the observations perfectly, while at an even higher metallicity $([\mathrm{Fe} / \mathrm{H}]=-0.8,47$ Tuc and $\mathrm{M} 71)$ they are again hotter by $50-100 \mathrm{~K}$. Given the uncertainty of the empirical determinations $( \pm 90 \mathrm{~K})$, this is a satisfactory agreement. We also compared our results to the latest $T_{\text {eff-values by Ferraro et al. (2006) for }}$ M 92, M 13, and 47 Tuc, given at three different values of $M_{\mathrm{bol}}$ along the RGB. After correcting for the slightly different distances, we find agreement within the errors given by Ferraro et al. (2006) for the two metal-poor clusters, but systematically hotter theoretical $T_{\text {eff }}$ for 47 Tuc $(\approx 150 \mathrm{~K})$, which are, however, only slightly outside the empirical uncertainties. Overall, we consider the agreement between our predicted effective temperatures and those determined from the infrared flux method satisfactory. Nonetheless, the fact that there is a tendency that the our values are too high renders colour-based age indicators more problematic than the vertical ones.

Finally, the RGB tip brightness can be compared to empirical data. We use Eq. (3) of Bellazzini et al. (2001), which gives the bolometric magnitude of the giant branch tip as a function of $[\mathrm{Fe} / \mathrm{H}]$. Inserting the corresponding numbers from Table 1, we find that the RGB tip of our isochrones (taken at $12 \mathrm{Gyr}$ ) is systematically too bright by 0.1 dex in luminosity, almost independent of metallicity. However, this discrepancy with the empirical values is very close to that found by Bellazzini et al. (2001) when comparing their results to the models by Salaris \& Cassisi (1998); it seems, therefore, that there is a systematic difference between theoretical and empirical values, which could be due the difficulty of finding the sparsely populated RGB tip.

The theoretical isochrones were transformed into the observational $M_{V}$ vs. $(B-V)_{0}$ plane using the transformations by Cassisi et al. (2004), which, however, do not include $\alpha$-enhancements in the theoretical atmosphere models. We always chose the transformation with $[\mathrm{Fe} / \mathrm{H}]_{\mathrm{atm}}=[\mathrm{M} / \mathrm{H}]_{\text {iso }}$, although this will render the transformed isochrones slightly too red on the RGB (Cassisi et al. 2004).

Alternatively, we tested the Yale transformations (Green 1988), which result in bluer RGBs than the Cassisi transformation. The influence on the determined ages was tested by generating a $10 \mathrm{Gyr}$ isochrone for $Z=0.001$ with the transformations by Cassisi et al. (2004), and then fitting it with isochrones using the Yale transformations. Depending on the age indicator, the transformations change the inferred age between $0.3 \mathrm{Gyr}$ (for TO-indicators) and 1.1 Gyr (indicators involving the bump). 


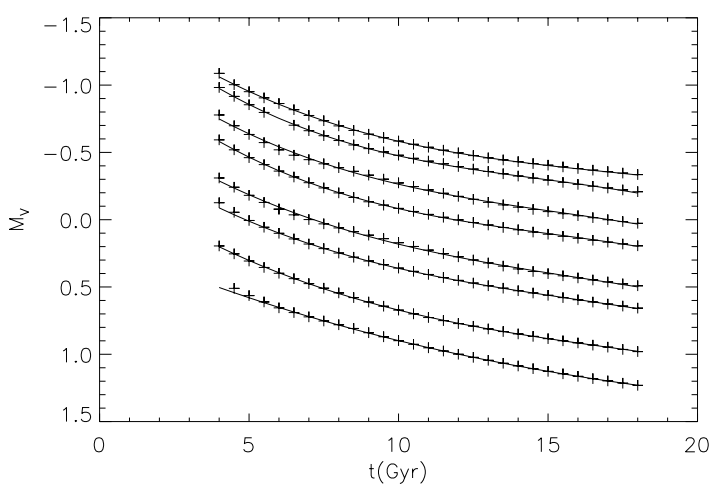

Fig. 9. $V_{\text {Bump }}$ as a function of age for all metallicities of Table 1 (crosses; increasing from bottom to top) and the third order polynomial fit (lines; see Table 8 ).

However, for $\Delta(B-V)_{\mathrm{TO}}^{\mathrm{RGB}}$ alone, the change is $+5 \mathrm{Gyr}$, when using the Yale transformations. Obviously, together with the hotter RGB effective temperature we find, the problematic colour transformations are another reason to consider colour-based age indicators less reliable, at least for absolute age determinations.

The theoretical luminosity function is obtained from the isochrone easily, as the mass along it is known, after folding it with an appropriate IMF. We choose an IMF exponent of $s=1.17$ (appropriate for globular cluster cores, see Zoccali \& Piotto 2000), with $s=3.35$ as the Salpeter value. The influence of $s$ is almost exclusively on the MS; since we need the LF only for the bump location, our results are almost completely independent of $s$.

\subsection{Theoretical values for age indicators}

The determination of the critical points along isochrones has been discussed before, or is straightforward. It is convenient to derive simple relations for their brightness and colour as function of age for all isochrone metallicities. An example ( $\left.V_{\text {Bump }}\right)$ is shown in Fig. 9. The analytical relations are obtained in all cases by fitting a third order polynomial through the data (Tables 8 and 9). No fitting of the metallicity dependence was done, however, for the sake of accuracy.

\section{Results}

\subsection{Method}

The general method is to calculate, for $n$ indicators and for each isochrone age, the value of $M(t)$ as defined by

$M(t)=\sum_{i=1}^{n} w_{i}\left(\frac{\Delta y_{i}-\widetilde{\Delta y}_{i}(t)}{\sigma\left(\Delta y_{i}\right)}\right)^{2}$,

where $\Delta y_{i}$ is the observed value of indicator $i, \widetilde{\Delta y_{i}}(t)$ the corresponding theoretical value for a particular isochrone age $t$, and $\sigma\left(\Delta y_{i}\right)$ the observational error (Brandt 1999). The $w_{i}$ are additional weights for the different indicators, which will hereafter be set to 0 or 1 only, but could take any value. The value of $\widetilde{\Delta y_{i}}(t)$ is computed from the polynomial fit and free of numerical errors. Systematic errors in the isochrones are not taken into account. Equation (3) resembles a $\chi^{2}$-test, but due to the unknown error statistics, we cannot claim that it really is one; rather, it is a goodness-of-fit measure. Following Press et al. (1992, Chap. 15.1), we introduce the quantity $Q$ as a measure
Table 2. Combinations of age indicators. The weight $w_{i}$ in Eq. (3) is given for each indicator.

\begin{tabular}{l|ccccccccc}
\hline \hline & \multicolumn{10}{|c}{ Indicator set } \\
Indicator & $\mathrm{A}$ & $\mathrm{B}$ & $\mathrm{C}$ & $\mathrm{D}$ & $\mathrm{E}$ & $\mathrm{F}$ & $\mathrm{BC}$ & $\mathrm{EF}$ & $\mathrm{S}$ \\
\hline$\Delta V_{\mathrm{TO}}^{\mathrm{ZAHB}}$ & 1 & 1 & 1 & 1 & 1 & 1 & 1 & 1 & 1 \\
$\Delta V_{\mathrm{TOb}}^{\mathrm{ZAHB}}$ & 1 & 1 & 1 & 1 & 1 & 1 & 1 & 1 & 1 \\
$\Delta V_{\mathrm{TOf}}^{\mathrm{ZAH}}$ & 1 & 1 & 1 & 1 & 1 & 1 & 1 & 1 & 1 \\
$\Delta V_{\mathrm{TO}}^{\text {Bump }}$ & 0 & 1 & 0 & 0 & 0 & 0 & 1 & 0 & 1 \\
$\Delta V_{\mathrm{ZAHp}}^{\text {Bump }}$ & 0 & 0 & 1 & 0 & 0 & 0 & 1 & 0 & 1 \\
$\Delta V_{\mathrm{TO}}^{\mathrm{tip}}$ & 0 & 0 & 0 & 1 & 0 & 0 & 0 & 0 & 1 \\
$\Delta(B-V)_{\mathrm{TO}}^{\mathrm{RGB}}$ & 0 & 0 & 0 & 0 & 1 & 0 & 0 & 1 & 1 \\
$\Delta(B-V)_{\mathrm{TO}}^{\mathrm{Bump}}$ & 0 & 0 & 0 & 0 & 0 & 1 & 0 & 1 & 1 \\
\hline
\end{tabular}

of the probability that the apparent discrepancies between data (age indicator values) and model (isochrone) are only by chance; that is, that the model is - in spite of the bad fit - a correct one. $Q=1-P$ is the complement of the probability $P$ that the model is the correct one. Mathematically, it is the incomplete gamma function depending on the number of degrees of freedom of the model and the value of $M$ (Press et al. 1992, Chapter 6). As recommended by Press et al. (1992), we chose $Q=0.001$ as the critical value, below which we reject models. This value is used as an acceptable measure, even for cases with presumably not normally distributed errors. Another requirement is that $M$ should be less than the number of degrees of freedom, which in our case are the 8 age indicators (equivalent to the normalised $\left.\chi^{2}<1\right)$.

Our best estimate for the age $t$ is then that of the isochrone with minimal $M(t)$, i.e., the age of the isochrone that appears to fit best all indicators simultaneously with equal weights assigned to them, and $Q>0.001$ implying that the remaining differences could be by chance due to the measurement errors. We recall (Sect. 4.3) that we always apply ischrones with the metallicity of the particular cluster; however, we do not refine the age grid of our isochrones, which implies an age resolution of 0.5 Gyr. A refined grid of isochrones or use of the polynomial fits of Tables 8 and 9 might possibly yield lower $M$-values than those given below. We have tested several cases with various combinations of age indicators (equivalent to setting the weights in Eq. (3) to 1); they are given in Table 2.

\subsection{A sample result: NGC 4833}

NGC 4833 is a cluster with $[\mathrm{Fe} / \mathrm{H}]_{\mathrm{CG}}=-1.71$; its CMD contains about 6700 stars and shows very well-defined structures. The HB is rather blue, but with a sufficient number of stars at $(B-V)_{\text {TO }}$ to determine the ZAHB.

Figure 10 shows $M(t)$ for various indicators. It is immediately clear that they do not agree at all. The three TO-related vertical indicators (Case A) yield an age of $13.5 \mathrm{Gyr}$ with $M(13.5)=2.6$ and $Q=0.457$, while $\Delta V_{\mathrm{TO}}^{\text {Bump }}$ has a sharp minimum at $8 \mathrm{Gyr}$, and $\Delta(B-V)_{\mathrm{TO}}^{\mathrm{RGB}}$ at $13.5 \mathrm{Gyr}$. Notice that $\Delta(B-V)_{\mathrm{TO}}^{\mathrm{Bump}}$ has an extremely flat curve at low $M$, which is due to the large error in this indicator. It provides, therefore, hardly any restriction on the global fit. Using all indicators (Case S) results in a minimum at $11.5 \mathrm{Gyr}$, which however, is pure coincidence, as $M(11.5)=191$ and $Q<0.001$. Clearly, $V_{\text {Bump }}$ is too low (bump too bright; it gets fainter with increasing age), which is also the reason why $\Delta V_{\mathrm{ZAHB}}^{\mathrm{Bump}}$ results in ages above the 


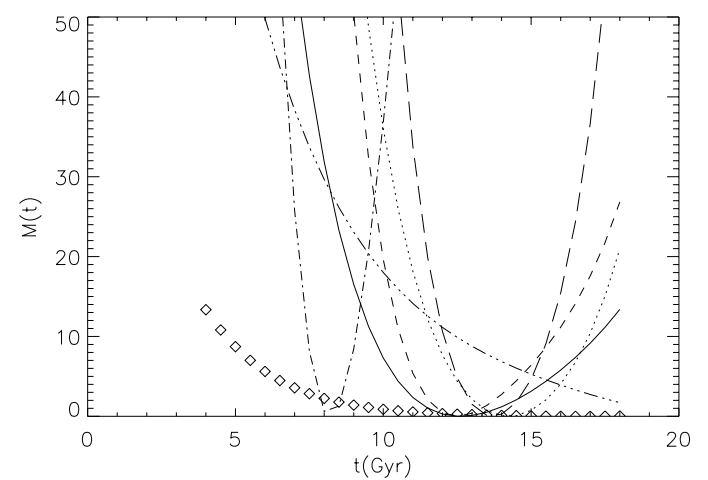

Fig. 10. $M(t)$ for various age indicators for the cluster NGC 4833; they are: $\Delta V_{\mathrm{TO}}^{\mathrm{ZAHB}}$ (solid), $\Delta V_{\mathrm{TOb}}^{\mathrm{ZAHB}}$ (dotted), $\Delta V_{\mathrm{TO}}^{\mathrm{ZAHB}}$ (dashed), $\Delta V_{\mathrm{TO}}^{\mathrm{Bump}}$ (dash dot), $\Delta V_{\mathrm{ZAHB}}^{\mathrm{Bump}}$ (dash dot dot dot), $\Delta(B-V)_{\mathrm{TO}}^{\mathrm{RGB}}$ (long dash), and $\Delta(B-V)_{\mathrm{TO}}^{\text {Bump }}$ (diamond symbols).

maximum age of $18 \mathrm{Gyr}$ for which we have isochrones. Since $V_{\text {Bump }}$ from the isochrones is too small, $\Delta V_{\mathrm{ZAHB}}^{\text {Bump }}$ has a too negative value for the actual age. To compensate, age has to be increased, and since the indicator is only weakly sensitive, the increase has to be substantial. The effect is in the opposite direction of that of $\Delta V_{\mathrm{TO}}^{\mathrm{Bump}}$, since the latter quantity has an opposite dependence on $V_{\text {Bump. }}$.

We add that $\Delta V_{\text {TO }}^{\text {tip }}$ yields an unrealistically low age of $4.5 \mathrm{Gyr}$, which is most likely due to an underestimate of the tip brightness in the data, but could also be due to errors in the cluster metallicity, as $\Delta V_{\mathrm{TO}}^{\mathrm{tip}}$ is sensitive to it.

\subsection{NGC 6934, a moderately metal-rich cluster}

Our second example is that of NGC $6934([\mathrm{Fe} / \mathrm{H}]=-1.30)$; the CMD contains close to 9500 stars and the HB is well populated. As in the previous case we see (Table 3) that the first three indicators agree very well and thus the "Case A" determination yields an age of $9.0 \mathrm{Gyr}$ with $Q=0.857$. Due to the higher number of stars, $V_{\text {tip }}$ can be determined more accurately and thus $\Delta V_{\text {TO }}^{\text {tip }}$ yields an age in agreement (Case D). In contrast, inclusion of the bump-brightness yields discrepant ages and Cases B and BC of Table 2 have a much lower confidence level (Fig. 11). The same is true when including the colour indicator $\Delta(B-V)_{\mathrm{TO}}^{\mathrm{RGB}}$ (Case $\left.\mathrm{E}\right)$, which requires a higher age. This implies that the RGB is too blue, relative to the TO, although our colour transformations have a systematic shift to the red (Sect. 4.3), and is a likely consequence of the theoretical effective temperatures. Cases $\mathrm{C}$ and $\mathrm{F}$ agree quite well with the turn-off indicators. Overall (Case $\mathrm{S}$ ) the age is $9.5 \mathrm{Gyr}$, but the confidence in this result is very low, and $M(9.5)=150$.

We have also investigated all other clusters obtaining consistent results: the bump in our isochrones appears to be too luminous, thereby leading to discrepant results when using $\Delta V_{\mathrm{TO}}^{\mathrm{Bump}}$ or $\Delta V_{\mathrm{ZAHB}}^{\mathrm{Bump}}$. The TO indicators give very consistent results with a very high confidence level, and the quality of $\Delta V_{\mathrm{TO}}^{\text {tip }}$ depends on the number of stars available for the location of the RGB tip, as should be expected.

\subsection{The special case of $M 15$}

We finally mention the peculiar case of M 15. Applying our method straightforwardly to the data by Piotto et al. (2002b), we
Table 3. Indicator value, age $t_{\min }$, minimum value $M\left(t_{\min }\right)$, and fit quality $Q$ for all age indicators applied to NGC 6934.

\begin{tabular}{lrrrr}
\hline \hline Indicator & Value & $t_{\min }$ & $M\left(t_{\min }\right)$ & $Q$ \\
\hline$\Delta V_{\mathrm{TO}}^{\mathrm{ZAHB}}$ & $3.24 \pm 0.08$ & 9.0 & 0.0840 & 0.772 \\
$\Delta V_{\mathrm{TOb}}^{\mathrm{ZAHB}}$ & $2.69 \pm 0.05$ & 9.5 & 0.0677 & 0.795 \\
$\Delta V_{\mathrm{TO}}^{\mathrm{ZHB}}$ & $4.13 \pm 0.06$ & 9.0 & 0.0044 & 0.947 \\
$\Delta V_{\mathrm{TO}}^{\mathrm{Bum}}$ & $3.62 \pm 0.06$ & 7.0 & 0.2170 & 0.641 \\
$\Delta V_{\mathrm{ZAHH}}^{\mathrm{Bump}}$ & $-0.38 \pm 0.05$ & 13.0 & 0.0030 & 0.956 \\
$\Delta V_{\mathrm{TO}}^{\mathrm{ti}}$ & $6.5 \pm 0.1$ & 8.0 & 0.0016 & 0.968 \\
$\Delta(B-V)_{\mathrm{TO}}^{\mathrm{RGB}}$ & $0.293 \pm 0.005$ & 12.0 & 0.0055 & 0.941 \\
$\Delta(B-V)_{\mathrm{TO}}^{\mathrm{Bump}}$ & $0.39 \pm 0.02$ & 17.0 & 0.0001 & 0.994 \\
\hline
\end{tabular}

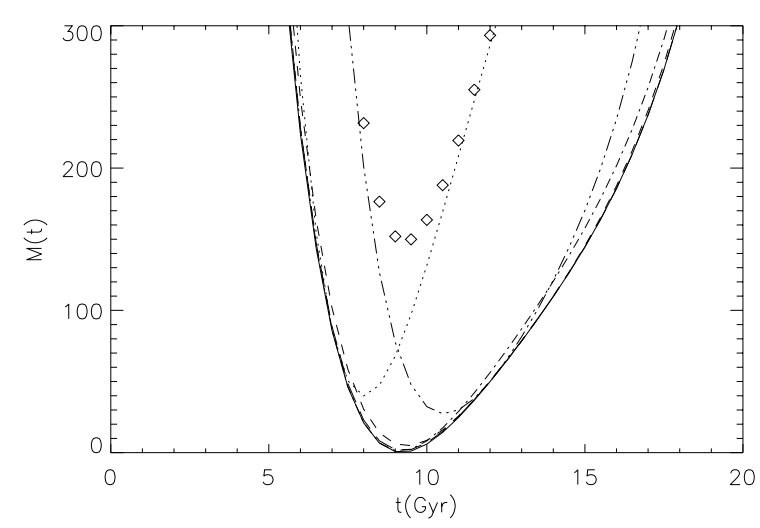

Fig. 11. Combinations of age indicators for NGC 6934: Case A (solid), Case B (dotted), Case C (dashed), Case D (dash dot), Case E (dash dot dot dot), and Case $\mathrm{S}$ (diamonds). Case $\mathrm{F}$ is not shown, as it coincides with Case A.

obtain around 9 Gyr only (see Table 5), with very low $Q$-values. The ZAHB is determined here at the TO-colour. Overlaying the resulting isochrone of 9 (Case A) and 11 Gyr (Case S) with the determined cluster ridge line aligned at the ZAHB (Fig. 12; upper panel), the corresponding distance modulus amounts to $15.32 \mathrm{mag}$. The figure shows that the isochrone is a very bad match to the CMD; it is too blue for all of the MS and too red on the RGB (one realizes how the "best" isochrone is a compromise match to all indicators). The inset, however, indicates that there might be a problem with the identification of the $\mathrm{HB}$, as the majority of stars is to the blue and at a higher brightness. Piotto (private communication) pointed out that the data were obtained in HST snapshot mode, which does not allow us to determine the mean brightness of RR Lyr variables, in which M 15 is very rich. Thus, a number of RR Lyr stars will be caught at minimum brightness. This was one reason for Recio-Blanco et al. (2005) to introduce the concept of template clusters. Identifying the blue part as the true HB, we obtain the results of the lower panel: our method yields 10.2-11.7 Gyr for Case A, and 11.5 Gyr for Case S; the ZAHB level corresponds to a distance modulus of $15.10 \mathrm{mag}$, and the $12 \mathrm{Gyr}$ isochrone matches the CMD quite well, except for being too blue on the lower main sequence.

As the photometry appears to be difficult to interpret, we used as an alternative that by Durrell \& Harris (1993). The ridge line we determine from this source agrees very well with that derived by Durrell and Harris themselves. Our age indicators now yield ages between 10.5 and 13.5 Gyr. The inferred distance modulus is 15.37 . From these data, the bump could not be located and the corresponding indicators cannot be used. The remaining indicators together yield $12.0 \mathrm{Gyr}$, in excellent agreement with the $11.7 \pm 0.8$ Gyr by Salaris \& Weiss (2002). Note 


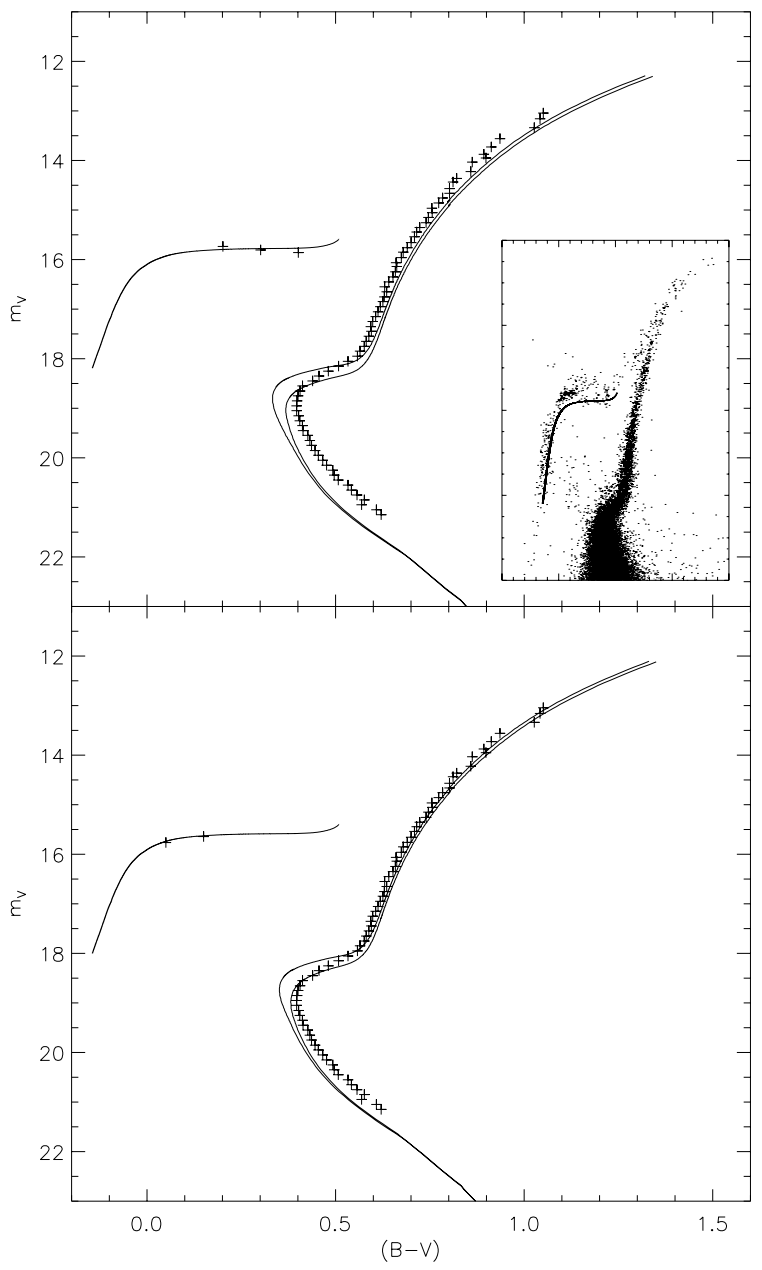

Fig. 12. Fit of the M 15 photometric data by Piotto et al. (2002b). In the upper panel our standard procedure was applied, fitting the HB at the TO colour. Isochrones of 9 and $11 \mathrm{Gyr}$ are overlaid. For the lower panel, the HB was fitted at the blue end, where the majority of stars is found, as can be seen in the inset of the upper panel. Here, the isochrone ages are 10 and 12 Gyr.

that the CMD of Durrell \& Harris (1993) does not show the splitting into a bluer, brighter and a redder, fainter part, as that by Piotto et al. (2002b) does (visible also on the original website dipastro .astro. unipd.it/globulars/databases). It is also less pronouned in the ground-based $(V-I)$ data by Rosenberg et al. (2000a). Whether the data by Piotto et al. (2002b) are erroneous, or our method of determining the ridge line fails in this case, or whether the core of M 15 indeed shows an anomalous CMD remains to be investigated. This case demonstrates that our method might also be capable of finding inconsistencies in the data.

\subsection{Quality of age indicators}

Figure 13 shows the quality of the Case A indicators' fits for all clusters investigated. We notice no correlation with metallicity and that the majority of cases have $Q>0.5$. In fact, all but three clusters (NGC 6093, 6642, and 6864) have $Q>0.001$, i.e., the best fit is unlikely to be just a chance result. This implies that our isochrones reproduce the critical TO region very well.

The situation is very different for Case B (Fig. 14), when we add $\Delta V_{\mathrm{TO}}^{\mathrm{Bump}}$ as an additional age indicator: no single cluster has $Q>0.1$ and 29 clusters are below our rejection value of 0.001 .

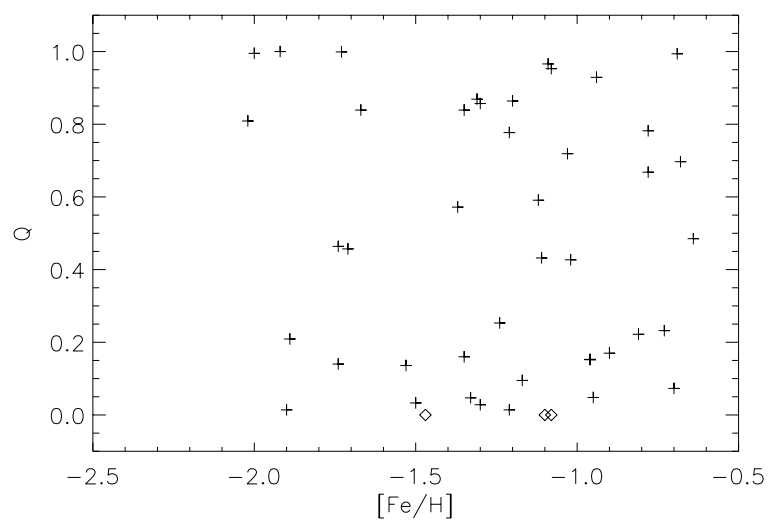

Fig. 13. Fit quality of Case A (TO-ZAHB-related) age indicators for all 46 clusters as a function of $[\mathrm{Fe} / \mathrm{H}]$. Diamonds indicate the clusters for which $Q<0.001$.

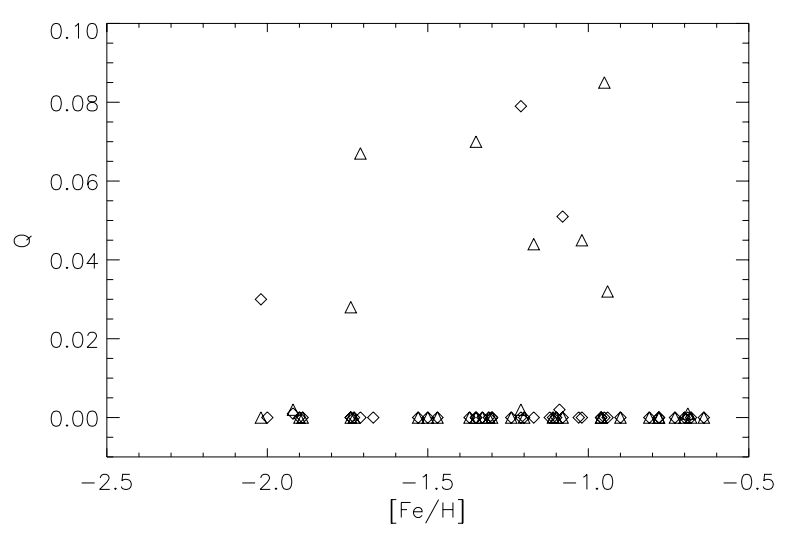

Fig. 14. Fit quality of Case B (Case A plus $\Delta V_{\text {TO }}^{\text {Bump }}$ ) age indicators for all 46 clusters as a function of $[\mathrm{Fe} / \mathrm{H}]$ (diamond symbols). Results for bump brightnesses corrected artificially by +0.25 mag are shown as triangles.

As the bump appears to be too bright by about $0.2-0.3$ mag, we artificially made it fainter by applying an ad hoc correction of $+0.25 \mathrm{mag}$, which is also indicated by Zoccali \& Piotto (2000). However, the result is basically the same (Fig. 14), such that an even larger correction would be needed. Indeed, with $0.35 \mathrm{mag}$, the ages obtained from $\Delta V_{\mathrm{TO}}^{\mathrm{Bump}}$ begin to be consistent with those of Case A. Note that adding $\Delta V_{\mathrm{ZAHB}}^{\mathrm{Bump}}$ to case $\mathrm{A}$ (i.e., Case $C$ ) does not degrade the fit quality significantly due to the rather shallow run of $M(t)$ for this indicator (Fig. 10).

The inclusion of the colour indicators (Cases E or F) results in no change of the fit quality at all because of the large errors for this indicator (Case F; see Sect. 5.2), or in a larger number of clusters to be rejected (Case $\mathrm{E}$, inclusion of $\left.\Delta(B-V)_{\mathrm{TO}}^{\mathrm{RGB}}\right)$ : 15 out of 46 clusters are below $Q=0.001$. Here, the theoretical uncertainty in the colour transformations is crucial and should be included in the calculation of $M$, although in this case the usefulness of $\Delta(B-V)_{\mathrm{TO}}^{\mathrm{RGB}}$ would just disappear. What is really needed are reliable and accurate transformations. Until then, vertical age indicators will remain the more reliable ones.

Finally, $\Delta V_{\mathrm{TO}}^{\mathrm{tip}}$ leads to systematically too low ages, as is expected from the problem of finding the true RGB tip in the observed CMD. Insteat, one should probably evaluate at what level the observed tip would be, given the number of stars observed in the uppermost bins of the CMD.

Table 5 contains the results for Case A, which is the most reliable one, and Case $\mathrm{S}$ for all clusters in comparison with the 

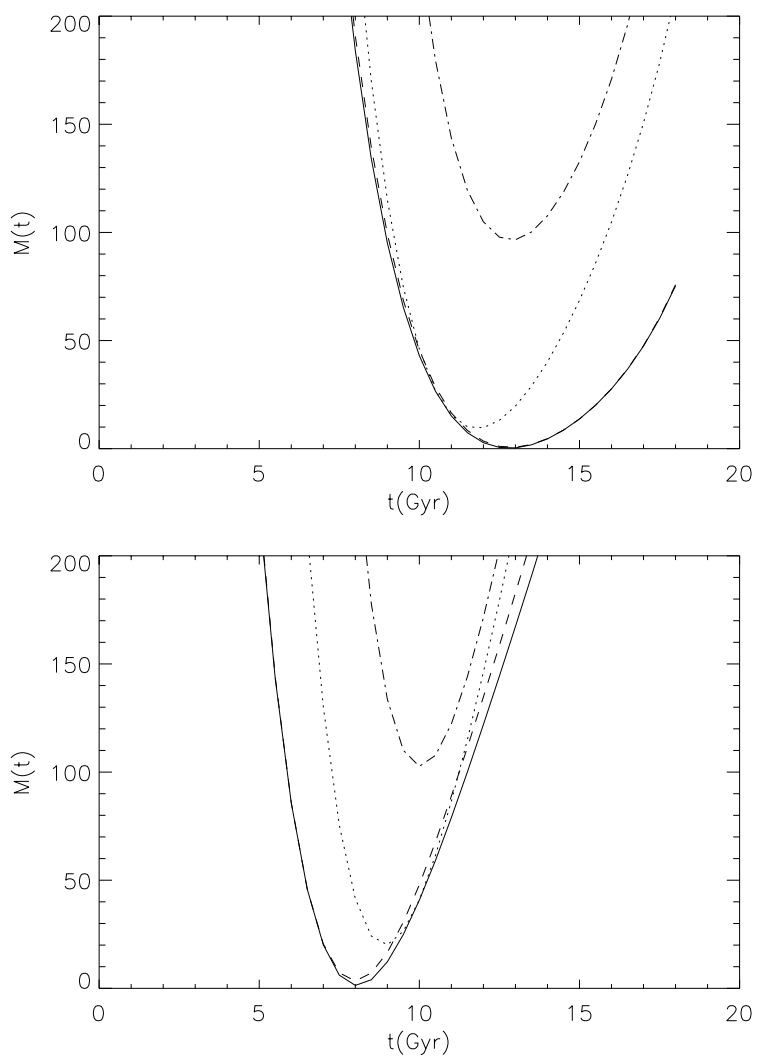

Fig. 15. Fitting functions for NGC 4833 (upper panel) and NGC 6934 (lower panel) using the BASTI (Pietrinferni et al. 2004) isochrones. The linetypes correspond to Case A (solid), B (dotted), C (dashed), and S (dash-dotted) in both cases.

ages found by Salaris \& Weiss (2002). If there is an age range given, it is that part of $M(t)$ with $Q>0.32$, which corresponds to the $1 \sigma$ range or a confidence level of $68 \%$. If only one age is listed, it is that where $M(t)$ has a minimum and where this minimum has a lower confidence level. This is particular true for all ages determined using all indicators (Case S). Note that very narrow age ranges ususally are not due to very sharp minima in $M(t)$, but due to a minimum value only slightly above the $1 \sigma$ level.

\subsection{Results for alternative isochrones}

The main conclusion of the preceding sections is that our bump brightnesses are too high. We have therefore looked into alternative sets of isochrones. Since Cassisi \& Salaris (1997) and Riello et al. (2003) claimed an improved agreement between observed and theoretical $V_{\text {Bump }}$ for their isochrones, we took the publically available BASTI $^{3}$ isochrones by Pietrinferni et al. (2004) and applied our method to them. A first inspection already confirmed that BASTI bumps are dimmer than ours by a few tenths of a magnitude. Figure 15 shows several fitting functions $M(t)$ for the two sample clusters NGC 4833 and NGC 6934 using the BASTI isochrones. Obviously, in these cases, the bump indicators are in much better agreement with those of set $\mathrm{A}$, and in addition, the remaining deviations are in both directions, that is, there are no systematic variations. The total fit (Case $S$ ) is still rather poor, but this is mainly due to the colour indicators, included here, too. Figure 16 illustrates the improvement

${ }^{3}$ URL: http://www.te.astro.it/BASTI

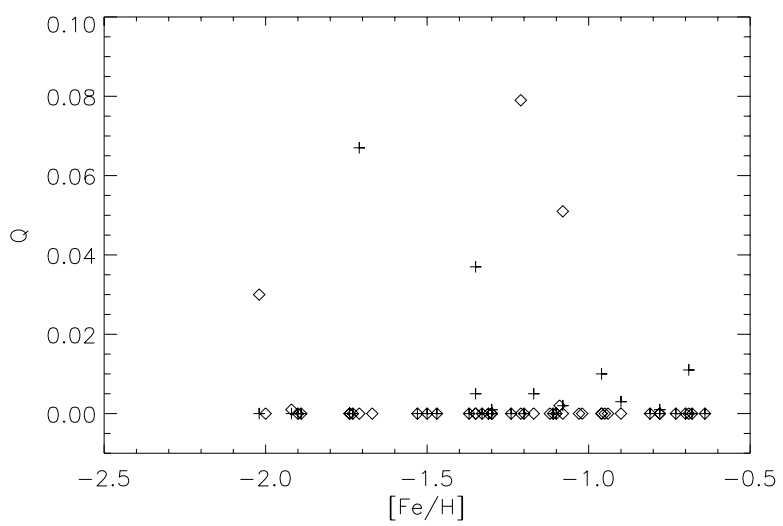

Fig. 16. Fit quality $Q$ for Case B indicators as a function of $[\mathrm{Fe} / \mathrm{H}]$ for both our own isochrones (diamonds) and those from Pietrinferni et al. (2004, crosses).

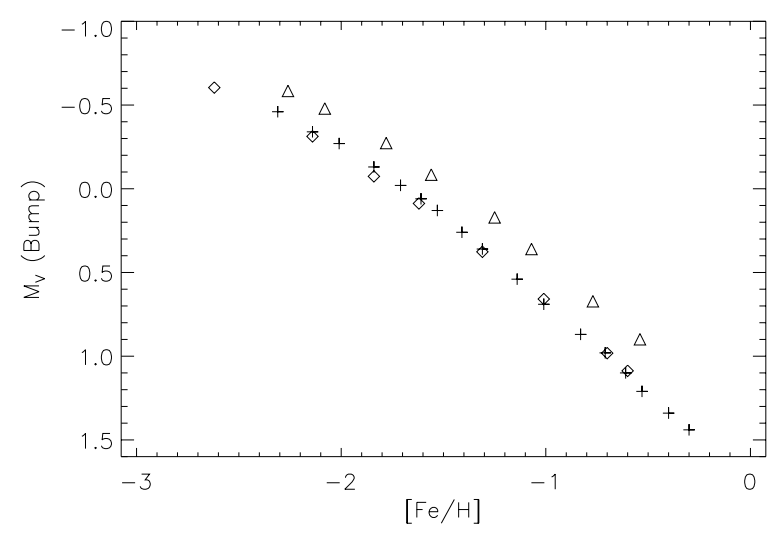

Fig. 17. Bump brightness for isochrones of $10 \mathrm{Gyr}$ as a function of metallicity $[\mathrm{Fe} / \mathrm{H}]$ for three sets of models: Bergbusch \& VandenBerg (2001, crosses), Pietrinferni et al. (2004, diamonds), and this paper (triangles).

Table 4. Comparison of bump properties for stellar models of $1 M_{\odot}$ and $Z=0.001$. The references are: Girardi et al. (2000, Padova), Cariulo et al. (2004, Pisa), Pietrinferni et al. (2004, BASTI), and Kim et al. $\left(2002, \mathrm{Y}^{2}\right)$.

\begin{tabular}{l|rrrrr}
\hline \hline & Padova & Pisa & BASTI & $\mathrm{Y}^{2}$ & This work \\
\hline Age (Gyr) & 6.514 & 6.331 & 6.023 & 6.769 & 6.222 \\
$\log L / L_{\odot}$ & 1.971 & 2.089 & 2.106 & 2.101 & 2.153 \\
$\log T_{\text {eff }}$ & 3.698 & 3.689 & 3.694 & 3.689 & 3.693 \\
Metal mix. & solar & solar & $\alpha$-enh. & $\alpha$-enh. & $\alpha$-enh. \\
Diffusion & no & yes & no & yes & yes \\
\hline
\end{tabular}

in $Q$ (Case B indicators) for at least 14 clusters when using the BASTI isochrones, and Table 5 contains the corresponding ages when considering both $\mathrm{TO}$ and bump indicators.

In Fig. 17 we show the bump location as a function of $[\mathrm{Fe} / \mathrm{H}]$ for our isochrones, that of BASTI, and those by Bergbusch \& VandenBerg (2001). Obviously, our bump location is systematically lower (brighter) than that of the other two sources. In Table 4 we compare bump locations and ages of $1 M_{\odot}$ stars with $Z=0.001$ for a number of authors, finding that there is quite a scatter in the bump properties. The Padova-bump has the lowest brightness due to the additional effect of overshooting from the lower convective boundary. The bump of our model is the brightest, and the age of BASTI's the youngest. We are currently comparing our RGB models in detail with those by Pietrinferni et al. (2004) to find out the reasons for the 
Table 5. Ages (in Gyr) for galactic globular clusters from Case A (Col. 4), from Case S (Col. 5) and quoted from Salaris \& Weiss (2002, Col., "SW02"). The age range is that for which $M(t)$ has $Q>0.32\left(1 \sigma\right.$-range). If only one age is given, it is $M_{\min }$, with $Q\left(M_{\min }\right)<0.32$. Columns 7 and 8 contain the result when using the BASTI isochrones (Pietrinferni et al. 2004) for Case A, and for case BC (see Table 2), which includes the bump brightness. Case $\mathrm{S}$ is not shown as it is not better than for our own isochrones.

\begin{tabular}{|c|c|c|c|c|c|c|c|}
\hline Name & & {$[\mathrm{Fe} / \mathrm{H}]$} & $t(\mathrm{~A})$ & $t_{\min }(\mathrm{S})$ & $t(\mathrm{SW} 02)$ & $t(\mathrm{~A})_{\mathrm{BASTI}}$ & $t(\mathrm{BC})_{\text {BASTI }}$ \\
\hline NGC 104 & 47 Tuc & -0.78 & $11.9-13.6$ & 11.5 & $10.7 \pm 1.0$ & $9.9-11.5$ & 9.5 \\
\hline NGC 362 & & -1.09 & $7.2-13.2$ & 8.5 & $8.7 \pm 1.5$ & $6.3-11.2$ & $6.8-10.8$ \\
\hline NGC 1261 & & -1.08 & $8.6-14.6$ & 9.5 & $8.6 \pm 1.1$ & $7.7-12.2$ & $10.5-10.6$ \\
\hline NGC 1851 & & -1.03 & 8.8-9.9 & 9.5 & $9.2 \pm 1.1$ & $7.9-8.7$ & 7.9-9.8 \\
\hline NGC 1904 & M 79 & -1.37 & $10.6-12.0$ & 11.0 & $11.7 \pm 1.3$ & $10.1-11.8$ & 12.0 \\
\hline NGC 2808 & & -1.11 & $9.4-10.0$ & 10.0 & $9.3 \pm 1.1$ & $8.4-8.6$ & 8.0 \\
\hline NGC 3201 & & -1.24 & 9.0 & 4.0 & $11.3 \pm 1.1$ & $7.3-8.8$ & 4.0 \\
\hline NGC 4147 & & -1.50 & 9.5 & 6.0 & - & $8.4-9.0$ & 4.0 \\
\hline NGC 4590 & M 68 & -2.00 & $8.5-11.0$ & 9.5 & $11.2 \pm 0.9$ & $9.2-12.5$ & 9.0 \\
\hline NGC 4833 & & -1.71 & $12.5-14.0$ & 11.5 & - & $12.1-14.2$ & 12.0 \\
\hline NGC 5024 & M 53 & -1.89 & 13.0 & 11.0 & - & $11.4-13.1$ & 10.0 \\
\hline NGC 5634 & & -1.61 & $8.4-10.1$ & 9.0 & - & $8.8-11.2$ & 7.5 \\
\hline NGC 5694 & & -1.74 & 12.5 & 13.0 & - & $11.2-13.0$ & 12.0 \\
\hline NGC 5824 & & -1.67 & $9.2-10.4$ & 11.0 & - & $8.8-9.5$ & 9.0 \\
\hline NGC 5904 & M 5 & -1.12 & $9.9-10.9$ & 11.0 & $10.9 \pm 1.1$ & $8.7-9.7$ & $8.5-9.6$ \\
\hline NGC 5927 & & -0.64 & $11.3-13.6$ & 8.0 & - & $8.6-10.4$ & 7.0 \\
\hline NGC 5986 & & -1.35 & 12.0 & 11.0 & - & 11.0 & 10.5 \\
\hline NGC 6093 & M 80 & -1.47 & 10.0 & 10.5 & $12.4 \pm 1.1$ & 9.0 & 8.0 \\
\hline NGC 6171 & M 107 & -0.95 & 9.0 & 9.5 & $11.7 \pm 0.8$ & $7.3-7.9$ & 7.5 \\
\hline NGC 6205 & M 13 & -1.33 & 11.0 & 15.5 & $11.9 \pm 1.1$ & $9.7-10.7$ & 10.5 \\
\hline NGC 6266 & M 62 & -1.02 & $11.0-12.1$ & 13.0 & - & 10.0 & 9.5 \\
\hline NGC 6273 & M 19 & -1.53 & $11.1-18.0$ & 15.0 & - & 17.0 & 17.0 \\
\hline NGC 6284 & & -1.17 & 11.0 & 11.0 & - & $8.9-10.0$ & 10.0 \\
\hline NGC 6287 & & -1.90 & 12.5 & 10.5 & - & $10.2-11.6$ & 9.0 \\
\hline NGC 6293 & & -1.73 & $7.9-8.8$ & 10.0 & - & $8.3-8.7$ & 8.5 \\
\hline NGC 6304 & & -0.68 & $12.3-14.9$ & 10.0 & - & $10.3-12.6$ & 9.0 \\
\hline NGC 6356 & & -0.69 & $12.0-18.0$ & 12.5 & - & $10.1-15.6$ & 10.0 \\
\hline NGC 6362 & & -0.96 & 10.5 & 9.0 & $11.0 \pm 1.3$ & $8.5-9.5$ & 8.5 \\
\hline NGC 6522 & & -1.21 & $13.9-16.1$ & 16.0 & - & $12.2-15.4$ & 13.0 \\
\hline NGC 6544 & & -1.20 & $7.1-8.5$ & 8.0 & - & $6.7-7.5$ & 7.0 \\
\hline NGC 6584 & & -1.30 & 9.0 & 10.5 & $11.3 \pm 1.4$ & $7.9-8.5$ & 8.0 \\
\hline NGC 6624 & & -0.70 & 12.0 & 10.5 & $10.6 \pm 1.4$ & 10.0 & 8.5 \\
\hline NGC 6637 & M 69 & -0.78 & $12.0-14.5$ & 11.5 & $10.6 \pm 1.4$ & 10.0 & 9.5 \\
\hline NGC 6638 & & -0.73 & 12.0 & 11.5 & - & $10.0-12.1$ & 10.0 \\
\hline NGC 6642 & & -1.08 & 10.0 & 10.0 & - & 8.5 & 8.5 \\
\hline NGC 6652 & & -0.81 & $11.5-11.8$ & 8.5 & $11.4 \pm 1.0$ & $9.1-10.8$ & 7.5 \\
\hline NGC 6681 & M 70 & -1.35 & $10.9-13.3$ & 9.5 & $11.5 \pm 1.4$ & $10.6-13.0$ & 9.5 \\
\hline NGC 6712 & & -0.94 & $10.2-14.7$ & 9.5 & $10.4 \pm 1.4$ & $8.7-12.1$ & 9.5 \\
\hline NGC 6723 & & -0.96 & 10.0 & 10.5 & $11.6 \pm 1.3$ & $8.3-9.5$ & 8.0 \\
\hline NGC 6838 & M 71 & -0.73 & 12.0 & 11.5 & $10.2 \pm 1.4$ & 10.0 & 10.0 \\
\hline NGC 6864 & M 75 & -1.10 & 9.0 & 9.5 & - & 8.0 & 7.5 \\
\hline NGC 6934 & & -1.30 & $8.7-10.2$ & 9.5 & $9.6 \pm 1.5$ & $7.8-8.7$ & 9.0 \\
\hline NGC 6981 & M 72 & -1.21 & 9.5 & 9.5 & - & $8.4-8.5$ & $7.9-8.5$ \\
\hline NGC 7078 & M 15 & -2.02 & $8.6-9.2$ & 11.0 & $11.7 \pm 0.8$ & 8.0 & 9.0 \\
\hline NGC 7089 & M 2 & -1.31 & $11.8-13.7$ & 12.5 & - & $10.9-11.6$ & 11.0 \\
\hline NGC 7099 & M 30 & -1.92 & $13.7-15.4$ & 13.0 & $11.9 \pm 1.4$ & $12.8-15.0$ & 14.0 \\
\hline
\end{tabular}

differences. At the present time, the scatter seems to be an intrinsic uncertainty in the various model calculations. Further evidence for the rather uncertain bump brightness comes from the comparison of a $0.9 M_{\odot} \operatorname{star}(Z=0.0001)$ by Straniero et al. (1997), recomputed by M. Limongi (private communication) with an updated version of his stellar evolution code: the luminosity of the new model agrees with that of our model within $\triangle \log L / L_{\odot}=0.009$, while the BASTI model is 0.058 dex fainter. However, all models of Straniero et al. (1997), which were used in Valenti et al. (2004) for comparison with infrared bump data, were consistently fainter than ours by $\approx 0.05$ dex.

If we use only the Case A indictors, the fit quality of the BASTI is very similar to that of our isochrones, but the ages are on average lower by about $0.5-1.5 \mathrm{Gyr}$.

\section{Discussion}

The aim of this work has been to attempt for the first time to simultaneously fit several age indicators of globular cluster CMDs. Such a more global approach removes a certain arbitrariness in the method of age determination and results in an overall best-fitting isochrone, which is not necessarily the one that best reproduces the TO. The bottom line of this paper is, however, that we are far from being able to do so. Instead, our method points to a number of inconsistencies and problems with our theoretical models. Apart from the case of M 15 (see Sect. 5.3), where the data deserve a second look by the observers, we doubt that a different data source would alter our findings. Nor did we modify the original data (Piotto et al. 2002b), for example, by changing the reddening. 
The most severe problem lies in the bump brightness, which, for our models consistently is too large by up to $0.3 \mathrm{mag}$. This may indicate the need for overshooting, which would result in a fainter bump. At the present time, however, it is evident that different theoretical models, which nominally all use up-todate physical input, differ widely in their predictions such that one first should look into code-specific reasons. We tested our method with the BASTI (Pietrinferni et al. 2004) isochrones, which show lower bump brightness, and found some improvement with respect to bump-related age indicators, but no final ages of consistently high confidence levels.

The second problem is the determination of the RGB tip brightness, which is due to the low number of stars observed in this phase. This leads to a consistent underestimate for the age derived from $\Delta V_{\mathrm{TO}}^{\mathrm{tip}}$, which could be cured by either applying a one-sided error-bar, or by a simulation of observations, or by modifying the age indicator, for example, by determining the level of the $10 \%$ brightest stars above the HB. Generally, however, this just leads to the assignment of an appreciable error range, which then would reduce the weight of that age indicator. This could also be done by assigning a lower weight $w_{i}$ in Eq. (3). One should mention in this context that theoretical models consistently appear to be brighter by $\approx 0.2$ mag than empirical determinations (Bellazzini et al. 2001, and Sect. 4.3).

Finally, the colour-related indicators on the RGB are unreliable due to the uncertainties of colour transformations and effective temperatures of the models. They, too, should receive a lower weight, or should be used for differential age determinations only, where the uncertainties are of less influence. The solution lies in better and calibrated transformations.

The most reliable age indicators appear to be those related to $V_{\mathrm{ZAHB}}$ and the three TO-brightness points. They give, for all cases, very consistent ages, and the confidence level of the solution is always very high. They are, within the errors, often in agreement with $\Delta V_{\mathrm{ZAHB}}^{\mathrm{Bump}}$ and $\Delta V_{\mathrm{TO}}^{\mathrm{tip}}$. Note that the overall best solution, even when omitting the most problematic indicators, does not yield the age of the classical indicator, $\Delta V_{\mathrm{TO}}^{\mathrm{ZAHB}}$, alone. This demonstrates that a global fit of cluster CMDs is indeed valuable.

Acknowledgements. We thank $\mathrm{H}$. Schlattl for help with the numerical calculations, M. Salaris for valuable comments on the manuscript, S. Cassisi and D. VandenBerg for making their models available to us, and M. Limongi for doing a separate new calculation. F. Meissner is grateful to the Max-Planck-Institut für Astrophysik for financial support. We also acknowledge the extremely careful and detailed report of the anonymous referee, which helped to significantly improve this paper.

\section{References}

Adelberger, E., Austin, S., Bahcall, J., et al. 1998, Rev. Mod. Phys., 70, 1265 Alexander, D., \& Ferguson, J. 1994, ApJ, 437, 879

Bellazzini, M., Ferraro, F., \& Pancino, E. 2001, ApJ, 556, 635

Bergbusch, P. A., \& VandenBerg, D. A. 1992, ApJS, 81, 163

Bergbusch, P. A., \& VandenBerg, D. A. 2001, ApJ, 556, 322

Brandt, S. 1999, Datenanalyse, 4th ed. (Spektrum Akademischer Verlag)

Buonanno, R., Corsi, C. E., Pulone, L., Fusi Pecci, F., \& Bellazzini, M. 1998, A\&A, 333, 505
Cariulo, P., Degl'Innocenti, S., \& Castellani, V. 2004, A\&A, 421, 1121 Carretta, E., \& Gratton, R. 1997, A\&AS, 121, 95

Carretta, E., Gratton, R., Clementini, G., \& Fusi Pecci, F. 2000, ApJ, 215

Carretta, E., Cohen, J. G., Gratton, R. G., \& Behr, B. B. 2001, AJ, 122, 1469

Cassisi, S., \& Salaris, M. 1997, MNRAS, 285, 593

Cassisi, S., Salaris, M., \& Irwin, A. 2003, ApJ, 588, 862

Cassisi, S., Salaris, M., Castelli, F., \& Pietrinferni, A. 2004, ApJ, 616, 498

Caughlan, G., \& Fowler, W. 1988, Atomic Data and Nuclear Data Tables, 40, 283

Caughlan, G., Fowler, W., Harris, H., \& Zimmerman, B. 1985, Atomic Data and Nuclear Data Tables, 32, 197

Chaboyer, B., Demarque, P., Kernan, P. J., Krauss, L. M., \& Sarajedini, A. 1996a, MNRAS, 283, 683

Chaboyer, B., Demarque, P., \& Sarajedini, A. 1996b, ApJ, 459, 558

Cho, D., Lee, S., Jeon, Y., \& Sim, K. 2005, AJ, 129, 1922

De Angeli, F., Piotto, G., Cassisi, S., et al. 2005, AJ, 130, 116

Durrell, P. R., \& Harris, W. E. 1993, AJ, 100, 1420

Eggleton, P. P., Faulkner, J., \& Flannery, B. P. 1973, MNRAS, 23, 325

Ferraro, F. R., Messineo, M., Fusi Pecci, F., et al. 1999, AJ, 118, 1738

Ferraro, F., Valenti, E., Straniero, O., \& Origlia, L. 2006, ApJ, 642, 225

Formicola, A., Imbriani, G., Costantini, H., et al. 2004, Phys. Lett. B, 591, 61

Frogel, J., Persson, S. E., \& Cohen, J. G. 1981, ApJ, 246, 842

Girardi, L., Bressan, A., Bertelli, G., \& Chiosi, C. 2000, A\&AS, 141, 371

Gratton, R. G., Fusi Pecci, F., Carretta, E., et al. 1997, ApJ, 491, 749

Green, E. M. 1988, in Calbration of Stellar Ages, ed. A. D. Philip (Schenectady,

N.Y.: L. Davis Press), 81

Grevesse, N., \& Noels, A. 1993, Physica Scripta, T47, 133

Hatzidimitriou, D. 1991, MNRAS, 251, 545

Iglesias, C., \& Rogers, F. 1996, ApJ, 464, 943

Junker, M., D’Alessandro, A., Zavatarelli, S., \& et al. 1998, Physical Review C, 57,2700

Kim, Y., Demarque, P., Yi, S., \& Alexander, D. 2002, ApJS, 143, 499

Meissner, F. 2005, Master's thesis, University of Munich

Pietrinferni, A., Cassisi, S., Salaris, M., \& Castelli, F. 2004, ApJ, 612, 168

Piotto, G., King, I. R., Djorgovski, S. G., et al. 2002a, A\&A, 391, 945

Piotto, G., King, I. R., Djorgovski, S. G., et al. 2002b, VizieR Online Data Catalog, 339, 10945

Press, W. H., Teukolsky, S. A., Vetterling, W. T., \& Flannery, B. P. 1992, Numerical Recipes in Fortran 77, 2nd ed., Vol. 1 (Cambridge University Press)

Recio-Blanco, A., Piotto, G., de Angeli, F., et al. 2005, A\&A, 432, 851

Renzini, A., \& Fusi Pecci, F. 1988, ARA\&A, 26, 199

Riello, M., Cassisi, S., Piotto, G., et al. 2003, A\&A, 410, 553

Rogers, F., Swenson, F., \& Iglesias, C. 1996, ApJ, 456, 902

Rosenberg, A., Saviane, I., Piotto, G., \& Aparicio, A. 1999, AJ, 118, 2306

Rosenberg, A., Aparicio, A., Savian, I., \& Piotto, G. 2000a, A\&AS, 145, 451

Rosenberg, A., Piotto, G., Saviane, I., \& Aparicio, A. 2000b, A\&AS, 144, 5

Rutledge, G. A., Hesser, J. E., \& Stetson, P. B. 1997, PASP, 109, 907

Salaris, M., \& Cassisi, S. 1997, MNRAS, 289, 406

Salaris, M., \& Cassisi, S. 1998, MNRAS, 298, 166

Salaris, M., \& Weiss, A. 1997, A\&A, 327, 107

Salaris, M., \& Weiss, A. 1998, A\&A, 335, 943

Salaris, M., \& Weiss, A. 2002, A\&A, 492

Salaris, M., Degl'Innocenti, S., \& Weiss, A. 1997, ApJ, 479, 665

Salaris, M., Cassisi, S., \& Weiss, A. 2002, PASP, 114, 375

Salpeter, E. 1955, ApJ, 121, 161

Sandquist, E. L., Bolte, M., Stetson, P. B., \& Hesser, J. E. 1996, ApJ, 470, 910

Saviane, I., Rosenberg, A., Piotto, G., \& Aparicio, A. 2000, A\&A, 355, 966

Serenelli, A., \& Weiss, A. 2005, A\&A, 442, 1041

Straniero, O., \& Chieffi, A. 1991, ApJS, 76, 525

Straniero, O., Chieffi, A., \& Limongi, M. 1997, ApJ, 490, 425

Thoul, A., Bahcall, J., \& Loeb, A. 1994, ApJ, 421, 828

Valenti, E., Ferraro, F. R., \& Origlia, L. 2004, MNRAS, 354, 815

VandenBerg, D. A. 2000, ApJS, 129, 315

Vandenberg, D. A., \& Durrell, P. R. 1990, AJ, 99, 221

VandenBerg, D. A., Bolte, M., \& Stetson, P. B. 1990, AJ, 100, 445

Weiss, A., \& Schlattl, H. 2000, A\&AS, 144, 487

Zinn, R., \& West, M. J. 1984, ApJS, 55, 45

Zoccali, M., \& Piotto, G. 2000, A\&A, 358, 943 
F. Meissner and A. Weiss: Global fitting of globular cluster age indicators, Online Material p 1

\section{Online Material}


F. Meissner and A. Weiss: Global fitting of globular cluster age indicators, Online Material p 2

Table 6. Data of the critical points of all Globular Cluster Colour-Magnitude Diagrams in our sample as determined by us (see Sect. 3); part I: the turn-off region and colours of turn-off, lower RGB, and bump.

\begin{tabular}{|c|c|c|c|c|c|c|c|c|}
\hline Name & & {$[\mathrm{Fe} / \mathrm{H}]$} & $V_{\mathrm{TO}}$ & $V_{\mathrm{TO}}^{\mathrm{b}}$ & $V_{\mathrm{TO}}^{\mathrm{f}}$ & $(B-V)_{\mathrm{TO}}$ & $(B-V)_{\mathrm{RGB}}$ & $(B-V)_{\text {Bump }}$ \\
\hline NGC 104 & 47 Tuc & -0.78 & $17.43 \pm 0.06$ & $17.06 \pm 0.01$ & $18.22 \pm 0.02$ & $0.502 \pm 0.001$ & $0.829 \pm 0.002$ & $0.89 \pm 0.02$ \\
\hline NGC 362 & & -1.09 & $18.56 \pm 0.07$ & $18.16 \pm 0.01$ & $19.51 \pm 0.02$ & $0.373 \pm 0.002$ & $0.703 \pm 0.003$ & $0.78 \pm 0.02$ \\
\hline NGC 1261 & & -1.08 & $20.15 \pm 0.08$ & $19.68 \pm 0.02$ & $21.15 \pm 0.02$ & $0.432 \pm 0.004$ & $0.753 \pm 0.004$ & $0.85 \pm 0.03$ \\
\hline NGC 1851 & & -1.03 & $19.41 \pm 0.07$ & $18.95 \pm 0.01$ & $20.32 \pm 0.02$ & $0.459 \pm 0.002$ & $0.783 \pm 0.003$ & $0.87 \pm 0.03$ \\
\hline NGC 1904 & M 79 & -1.37 & $19.58 \pm 0.07$ & $19.10 \pm 0.01$ & $20.47 \pm 0.02$ & $0.441 \pm 0.003$ & $0.706 \pm 0.003$ & $0.88 \pm 0.03$ \\
\hline NGC 2808 & & -1.11 & $18.86 \pm 0.06$ & $18.42 \pm 0.01$ & $19.82 \pm 0.02$ & $0.396 \pm 0.002$ & $0.707 \pm 0.002$ & $0.78 \pm 0.03$ \\
\hline NGC 3201 & & -1.24 & $17.41 \pm 0.08$ & $17.02 \pm 0.04$ & $18.23 \pm 0.03$ & $0.493 \pm 0.003$ & $0.790 \pm 0.010$ & $0.74 \pm 0.03$ \\
\hline NGC 4147 & & -1.50 & $20.32 \pm 0.07$ & $19.84 \pm 0.02$ & $21.07 \pm 0.04$ & $0.401 \pm 0.004$ & $0.659 \pm 0.008$ & $0.65 \pm 0.03$ \\
\hline NGC 4590 & M 68 & -2.00 & $18.92 \pm 0.09$ & $18.39 \pm 0.03$ & $19.82 \pm 0.03$ & $0.382 \pm 0.003$ & $0.644 \pm 0.006$ & $0.73 \pm 0.02$ \\
\hline NGC 4833 & & -1.71 & $18.05 \pm 0.09$ & $17.57 \pm 0.02$ & $18.96 \pm 0.02$ & $0.407 \pm 0.003$ & $0.672 \pm 0.004$ & $0.79 \pm 0.03$ \\
\hline NGC 5024 & M 53 & -1.89 & $20.23 \pm 0.08$ & $19.72 \pm 0.02$ & $21.10 \pm 0.02$ & $0.389 \pm 0.002$ & $0.657 \pm 0.002$ & $0.75 \pm 0.03$ \\
\hline NGC 5634 & & -1.61 & $20.91 \pm 0.09$ & $20.46 \pm 0.02$ & $21.64 \pm 0.03$ & $0.369 \pm 0.003$ & $0.634 \pm 0.003$ & $0.71 \pm 0.02$ \\
\hline NGC 5694 & & -1.74 & $21.65 \pm 0.07$ & $21.19 \pm 0.02$ & $22.52 \pm 0.03$ & $0.424 \pm 0.003$ & $0.683 \pm 0.004$ & - \\
\hline NGC 5824 & & -1.67 & $21.51 \pm 0.06$ & $20.98 \pm 0.02$ & $22.42 \pm 0.03$ & $0.395 \pm 0.002$ & $0.659 \pm 0.002$ & $0.76 \pm 0.02$ \\
\hline NGC 5904 & M 5 & -1.12 & $18.35 \pm 0.06$ & $17.90 \pm 0.02$ & $19.25 \pm 0.03$ & $0.431 \pm 0.001$ & $0.732 \pm 0.003$ & $0.81 \pm 0.03$ \\
\hline NGC 5927 & & -0.64 & $18.73 \pm 0.07$ & $18.43 \pm 0.01$ & $19.50 \pm 0.02$ & $0.575 \pm 0.003$ & $0.954 \pm 0.007$ & $0.99 \pm 0.03$ \\
\hline NGC 5986 & & -1.35 & $19.25 \pm 0.08$ & $18.75 \pm 0.01$ & $20.30 \pm 0.02$ & $0.447 \pm 0.003$ & $0.727 \pm 0.003$ & $0.82 \pm 0.02$ \\
\hline NGC 6093 & M 80 & -1.47 & $18.90 \pm 0.06$ & $18.53 \pm 0.02$ & $19.38 \pm 0.02$ & $0.579 \pm 0.005$ & $0.774 \pm 0.004$ & $0.85 \pm 0.02$ \\
\hline NGC 6171 & M 107 & -0.95 & $18.14 \pm 0.07$ & $17.74 \pm 0.02$ & $18.92 \pm 0.04$ & $0.650 \pm 0.004$ & $0.944 \pm 0.008$ & $1.02 \pm 0.04$ \\
\hline NGC 6205 & M 13 & -1.33 & $18.33 \pm 0.07$ & $17.89 \pm 0.01$ & $19.07 \pm 0.03$ & $0.471 \pm 0.003$ & $0.719 \pm 0.003$ & - \\
\hline NGC 6266 & M 62 & -1.02 & $18.21 \pm 0.07$ & $17.77 \pm 0.02$ & $19.15 \pm 0.02$ & $0.522 \pm 0.002$ & $0.805 \pm 0.003$ & $0.89 \pm 0.03$ \\
\hline NGC 6273 & M 19 & -1.53 & $18.71 \pm 0.07$ & $18.16 \pm 0.02$ & $19.70 \pm 0.02$ & $0.465 \pm 0.003$ & $0.729 \pm 0.004$ & - \\
\hline NGC 6284 & & -1.17 & $19.92 \pm 0.07$ & $19.48 \pm 0.02$ & $20.71 \pm 0.02$ & $0.502 \pm 0.003$ & $0.774 \pm 0.005$ & $0.88 \pm 0.02$ \\
\hline NGC 6287 & & -1.90 & $18.58 \pm 0.08$ & $0 \pm 0.04$ & $19.33 \pm 0.03$ & $0.533 \pm 0.006$ & $0.776 \pm 0.009$ & $0.78 \pm 0.05$ \\
\hline NGC 6293 & & -1.73 & $18.62 \pm 0.07$ & $18.12 \pm 0.02$ & $19.54 \pm 0.03$ & $0.365 \pm 0.002$ & $0.631 \pm 0.006$ & - \\
\hline NGC 6304 & & -0.68 & $18.24 \pm 0.06$ & $17.91 \pm 0.01$ & $18.98 \pm 0.02$ & $0.542 \pm 0.004$ & $0.898 \pm 0.005$ & $0.93 \pm 0.03$ \\
\hline NGC 6356 & & -0.69 & $20.26 \pm 0.09$ & $19.88 \pm 0.01$ & $20.96 \pm 0.02$ & $0.547 \pm 0.003$ & $0.875 \pm 0.003$ & $0.94 \pm 0.04$ \\
\hline NGC 6362 & & -0.96 & $18.50 \pm 0.06$ & $18.12 \pm 0.02$ & $19.30 \pm 0.04$ & $0.488 \pm 0.002$ & $0.794 \pm 0.007$ & $0.88 \pm 0.02$ \\
\hline NGC 6522 & & -1.21 & $18.94 \pm 0.09$ & $18.27 \pm 0.04$ & $19.63 \pm 0.02$ & $0.599 \pm 0.005$ & $0.820 \pm 0.020$ & $0.90 \pm 0.20$ \\
\hline NGC 6544 & & -1.20 & $16.21 \pm 0.11$ & $15.76 \pm 0.02$ & $17.22 \pm 0.03$ & $0.535 \pm 0.009$ & $0.830 \pm 0.020$ & - \\
\hline NGC 6584 & & -1.30 & $19.49 \pm 0.07$ & $19.09 \pm 0.02$ & $20.34 \pm 0.03$ & $0.420 \pm 0.002$ & $0.705 \pm 0.006$ & - \\
\hline NGC 6624 & & -0.70 & $18.73 \pm 0.06$ & $18.45 \pm 0.02$ & $19.47 \pm 0.04$ & $0.571 \pm 0.003$ & $0.901 \pm 0.005$ & $0.94 \pm 0.03$ \\
\hline NGC 6637 & M 69 & -0.78 & $19.02 \pm 0.08$ & $18.65 \pm 0.01$ & $19.86 \pm 0.02$ & $0.541 \pm 0.003$ & $0.867 \pm 0.004$ & $0.93 \pm 0.03$ \\
\hline NGC 6638 & & -0.73 & $19.06 \pm 0.07$ & $18.67 \pm 0.02$ & $20.04 \pm 0.02$ & $0.532 \pm 0.004$ & $0.810 \pm 0.010$ & $0.88 \pm 0.05$ \\
\hline NGC 6642 & & -1.08 & $18.64 \pm 0.09$ & $18.33 \pm 0.03$ & $19.32 \pm 0.02$ & $0.524 \pm 0.008$ & $0.760 \pm 0.040$ & $0.90 \pm 0.20$ \\
\hline NGC 6652 & & -0.81 & $19.17 \pm 0.07$ & $18.87 \pm 0.02$ & $19.95 \pm 0.03$ & $0.535 \pm 0.004$ & $0.876 \pm 0.005$ & $0.92 \pm 0.02$ \\
\hline NGC 6681 & M 70 & -1.35 & $18.93 \pm 0.08$ & $18.46 \pm 0.02$ & $19.83 \pm 0.02$ & $0.455 \pm 0.002$ & $0.736 \pm 0.004$ & $0.82 \pm 0.02$ \\
\hline NGC 6712 & & -0.94 & $18.35 \pm 0.08$ & $17.93 \pm 0.02$ & $19.08 \pm 0.03$ & $0.494 \pm 0.004$ & $0.818 \pm 0.006$ & $0.90 \pm 0.05$ \\
\hline NGC 6723 & & -0.96 & $18.73 \pm 0.08$ & $18.37 \pm 0.01$ & $19.59 \pm 0.03$ & $0.513 \pm 0.002$ & $0.813 \pm 0.003$ & $0.87 \pm 0.02$ \\
\hline NGC 6838 & M 71 & -0.73 & $17.22 \pm 0.08$ & $16.86 \pm 0.02$ & $17.87 \pm 0.03$ & $0.521 \pm 0.007$ & $0.870 \pm 0.010$ & - \\
\hline NGC 6864 & M 75 & -1.10 & $20.40 \pm 0.09$ & $20.07 \pm 0.02$ & $21.11 \pm 0.02$ & $0.479 \pm 0.003$ & $0.792 \pm 0.003$ & $0.87 \pm 0.03$ \\
\hline NGC 6934 & & -1.30 & $20.07 \pm 0.06$ & $19.51 \pm 0.02$ & $20.95 \pm 0.03$ & $0.424 \pm 0.002$ & $0.717 \pm 0.005$ & $0.81 \pm 0.02$ \\
\hline NGC 6981 & M 72 & -1.21 & $20.01 \pm 0.07$ & $19.65 \pm 0.02$ & $20.86 \pm 0.03$ & $0.414 \pm 0.003$ & $0.704 \pm 0.004$ & $0.77 \pm 0.02$ \\
\hline NGC 7078 & M 15 & -2.02 & $18.92 \pm 0.07$ & $18.39 \pm 0.01$ & $19.85 \pm 0.02$ & $0.396 \pm 0.002$ & $0.647 \pm 0.002$ & $0.76 \pm 0.02$ \\
\hline NGC 7089 & M 2 & -1.31 & $19.30 \pm 0.07$ & $18.77 \pm 0.01$ & $20.22 \pm 0.02$ & $0.387 \pm 0.003$ & $0.666 \pm 0.004$ & - \\
\hline NGC 7099 & M 30 & -1.92 & $18.64 \pm 0.08$ & $18.07 \pm 0.02$ & $19.50 \pm 0.04$ & $0.407 \pm 0.002$ & $0.672 \pm 0.004$ & - \\
\hline
\end{tabular}


F. Meissner and A. Weiss: Global fitting of globular cluster age indicators, Online Material p 3

Table 7. As Table 6, but displaying horizontal branch, RGB bump, and tip brightness as well as the cluster distances inferred from $V_{\text {ZAHB }}$ and $V_{\text {tip }}$. $(B-V)_{\mathrm{ZAHB}}$ is the colour of the point on the ZAHB closest to the TO colour.

\begin{tabular}{|c|c|c|c|c|c|c|}
\hline Name & $V_{\mathrm{ZAHB}}$ & $(m-M)_{\mathrm{ZAHB}}$ & $V_{\text {tip }}$ & $(m-M)_{\text {tip }}$ & $V_{\text {Bump }}$ & $(B-V)_{\mathrm{ZAHB}}$ \\
\hline NGC 104 & $13.94 \pm 0.06$ & $13.24 \pm 0.03$ & $11.39 \pm 0.04$ & $13.6 \pm 0.1$ & 14.30 & 0.626 \\
\hline NGC 362 & $15.34 \pm 0.05$ & $14.66 \pm 0.03$ & $12.19 \pm 0.09$ & $14.8 \pm 0.1$ & 15.26 & 0.383 \\
\hline NGC 1261 & $16.80 \pm 0.20$ & $16.09 \pm 0.03$ & $13.80 \pm 0.10$ & $16.5 \pm 0.1$ & 16.62 & 0.451 \\
\hline NGC 1851 & $16.18 \pm 0.05$ & $15.48 \pm 0.02$ & $13.16 \pm 0.05$ & $15.7 \pm 0.1$ & 16.05 & 0.459 \\
\hline NGC 1904 & $16.16 \pm 0.05$ & $15.55 \pm 0.04$ & $13.20 \pm 0.10$ & $16.0 \pm 0.1$ & 15.24 & 0.249 \\
\hline NGC 2808 & $15.63 \pm 0.05$ & $15.03 \pm 0.03$ & $12.67 \pm 0.02$ & $15.3 \pm 0.1$ & 15.60 & 0.396 \\
\hline NGC 3201 & $14.22 \pm 0.09$ & $13.66 \pm 0.04$ & $13.00 \pm 0.10$ & $15.7 \pm 0.1$ & 15.72 & 0.165 \\
\hline NGC 4147 & $17.06 \pm 0.05$ & $16.39 \pm 0.03$ & $13.80 \pm 0.40$ & $16.7 \pm 0.4$ & 18.11 & 0.401 \\
\hline NGC 4590 & $15.70 \pm 0.10$ & $15.25 \pm 0.04$ & $12.40 \pm 0.50$ & $15.4 \pm 0.4$ & 15.07 & 0.107 \\
\hline NGC 4833 & $14.52 \pm 0.05$ & $13.95 \pm 0.04$ & $12.20 \pm 0.10$ & $15.2 \pm 0.1$ & 14.18 & 0.220 \\
\hline NGC 5024 & $16.70 \pm 0.05$ & $16.30 \pm 0.01$ & $13.67 \pm 0.08$ & $16.7 \pm 0.1$ & - & 0.389 \\
\hline NGC 5634 & $17.70 \pm 0.10$ & $17.15 \pm 0.04$ & $14.10 \pm 0.20$ & $17.1 \pm 0.2$ & 17.28 & 0.100 \\
\hline NGC 5694 & $18.16 \pm 0.05$ & $17.89 \pm 0.03$ & $15.25 \pm 0.08$ & $18.2 \pm 0.1$ & - & 0.424 \\
\hline NGC 5824 & $18.28 \pm 0.05$ & $17.59 \pm 0.01$ & $14.90 \pm 0.04$ & $17.8 \pm 0.1$ & 17.80 & 0.395 \\
\hline NGC 5904 & $15.02 \pm 0.05$ & $14.35 \pm 0.03$ & $12.22 \pm 0.08$ & $14.9 \pm 0.1$ & 14.90 & 0.431 \\
\hline NGC 5927 & $15.28 \pm 0.08$ & $15.00 \pm 0.03$ & $13.24 \pm 0.05$ & $15.2 \pm 0.2$ & 15.90 & 0.856 \\
\hline NGC 5986 & $15.82 \pm 0.05$ & $15.26 \pm 0.03$ & $12.59 \pm 0.08$ & $15.4 \pm 0.1$ & 15.58 & 0.447 \\
\hline NGC 6093 & $15.49 \pm 0.05$ & $15.30 \pm 0.03$ & $12.55 \pm 0.07$ & $15.4 \pm 0.1$ & 15.40 & 0.579 \\
\hline NGC 6171 & $14.95 \pm 0.05$ & $14.34 \pm 0.03$ & $12.30 \pm 0.20$ & $14.8 \pm 0.2$ & 14.83 & 0.650 \\
\hline NGC 6205 & $14.90 \pm 0.07$ & $14.38 \pm 0.03$ & $11.80 \pm 0.10$ & $14.6 \pm 0.1$ & - & 0.125 \\
\hline NGC 6266 & $14.81 \pm 0.05$ & $14.18 \pm 0.02$ & $12.03 \pm 0.04$ & $14.6 \pm 0.1$ & 14.82 & 0.525 \\
\hline NGC 6273 & $14.89 \pm 0.05$ & $14.74 \pm 0.03$ & $12.04 \pm 0.05$ & $14.9 \pm 0.1$ & - & 0.465 \\
\hline NGC 6284 & $16.52 \pm 0.05$ & $15.99 \pm 0.03$ & $13.60 \pm 0.10$ & $16.3 \pm 0.1$ & 16.30 & 0.502 \\
\hline NGC 6287 & $15.02 \pm 0.05$ & $15.00 \pm 0.02$ & $12.40 \pm 0.20$ & $15.4 \pm 0.2$ & 15.98 & 0.533 \\
\hline NGC 6293 & $15.63 \pm 0.05$ & $14.85 \pm 0.04$ & $12.20 \pm 0.10$ & $15.2 \pm 0.1$ & - & 0.100 \\
\hline NGC 6304 & $14.69 \pm 0.08$ & $14.22 \pm 0.01$ & $12.81 \pm 0.08$ & $14.9 \pm 0.2$ & 15.40 & 0.778 \\
\hline NGC 6356 & $16.60 \pm 0.20$ & $15.99 \pm 0.02$ & $14.07 \pm 0.05$ & $16.2 \pm 0.2$ & 17.19 & 0.585 \\
\hline NGC 6362 & $15.14 \pm 0.05$ & $14.20 \pm 0.02$ & $12.60 \pm 0.30$ & $15.0 \pm 0.3$ & 15.23 & 0.488 \\
\hline NGC 6522 & $15.14 \pm 0.05$ & $14.63 \pm 0.03$ & $12.09 \pm 0.09$ & $14.8 \pm 0.1$ & 15.63 & 0.599 \\
\hline NGC 6544 & $13.21 \pm 0.07$ & $12.33 \pm 0.04$ & $11.00 \pm 0.20$ & $13.7 \pm 0.2$ & - & 0.100 \\
\hline NGC 6584 & $16.29 \pm 0.05$ & $15.61 \pm 0.03$ & $13.30 \pm 0.20$ & $16.1 \pm 0.2$ & - & 0.420 \\
\hline NGC 6624 & $15.32 \pm 0.05$ & $14.74 \pm 0.02$ & $12.99 \pm 0.08$ & $15.1 \pm 0.2$ & 15.83 & 0.700 \\
\hline NGC 6637 & $15.52 \pm 0.07$ & $14.82 \pm 0.03$ & $13.03 \pm 0.07$ & $15.3 \pm 0.1$ & 15.90 & 0.726 \\
\hline NGC 6638 & $15.69 \pm 0.05$ & $14.91 \pm 0.01$ & $13.09 \pm 0.06$ & $15.5 \pm 0.1$ & 15.89 & 0.532 \\
\hline NGC 6642 & $15.34 \pm 0.15$ & $14.72 \pm 0.03$ & $12.70 \pm 0.10$ & $15.3 \pm 0.1$ & 15.39 & 0.524 \\
\hline NGC 6652 & $15.78 \pm 0.08$ & $15.14 \pm 0.04$ & $13.30 \pm 0.10$ & $15.5 \pm 0.2$ & 16.15 & 0.750 \\
\hline NGC 6681 & $15.47 \pm 0.07$ & $14.95 \pm 0.02$ & $12.60 \pm 0.20$ & $15.4 \pm 0.2$ & 15.37 & 0.175 \\
\hline NGC 6712 & $14.90 \pm 0.10$ & $14.41 \pm 0.03$ & $13.18 \pm 0.09$ & $15.7 \pm 0.1$ & 15.11 & 0.545 \\
\hline NGC 6723 & $15.47 \pm 0.05$ & $14.91 \pm 0.03$ & $13.20 \pm 0.10$ & $15.7 \pm 0.1$ & 15.50 & 0.513 \\
\hline NGC 6838 & $13.73 \pm 0.07$ & $13.33 \pm 0.04$ & $12.30 \pm 0.30$ & $14.5 \pm 0.3$ & - & 0.697 \\
\hline NGC 6864 & $17.17 \pm 0.05$ & $16.65 \pm 0.02$ & $14.35 \pm 0.05$ & $17.0 \pm 0.1$ & 17.19 & 0.479 \\
\hline NGC 6934 & $16.81 \pm 0.05$ & $16.24 \pm 0.04$ & $13.50 \pm 0.10$ & $16.3 \pm 0.1$ & 16.44 & 0.155 \\
\hline NGC 6981 & $16.81 \pm 0.05$ & $16.03 \pm 0.02$ & $13.80 \pm 0.20$ & $16.5 \pm 0.2$ & 16.57 & 0.414 \\
\hline NGC 7078 & $15.86 \pm 0.05$ & $15.32 \pm 0.03$ & $12.42 \pm 0.07$ & $15.5 \pm 0.8$ & 14.97 & 0.396 \\
\hline NGC 7089 & $15.77 \pm 0.05$ & $15.05 \pm 0.02$ & $13.06 \pm 0.08$ & $15.8 \pm 0.1$ & - & 0.225 \\
\hline NGC 7099 & $14.99 \pm 0.05$ & $14.69 \pm 0.04$ & $12.80 \pm 0.10$ & $15.8 \pm 0.1$ & - & 0.336 \\
\hline
\end{tabular}


F. Meissner and A. Weiss: Global fitting of globular cluster age indicators, Online Material p 4

Table 8. Polynomial relations for points along our isochrones used as age indicators. The relations are all of the form $f(t)=a_{0}+a_{1} t+a_{2} t^{2}+$ $a_{3} t^{3}$, where $t$ is in Gyr. Part I: $V$-magnitudes.

\begin{tabular}{|c|c|c|c|c|}
\hline$[\mathrm{Fe} / \mathrm{H}]$ & $a_{0}$ & $a_{1}$ & $a_{2}$ & $a_{3}$ \\
\hline \multicolumn{5}{|c|}{$V_{\text {TO }}$} \\
\hline-2.26 & 1.081588 & 0.409828 & -0.019137 & 0.000357 \\
\hline-2.08 & 1.108813 & 0.416247 & -0.019509 & 0.000357 \\
\hline-1.78 & 1.100272 & 0.458946 & -0.023650 & 0.000468 \\
\hline-1.56 & 1.155703 & 0.478126 & -0.026089 & 0.000546 \\
\hline-1.25 & 1.217895 & 0.527360 & -0.032193 & 0.000736 \\
\hline-1.07 & 1.128014 & 0.589114 & -0.038899 & 0.000941 \\
\hline-0.77 & 2.053031 & 0.376031 & -0.021472 & 0.000476 \\
\hline-0.54 & 2.557615 & 0.261690 & -0.012317 & 0.000242 \\
\hline \multicolumn{5}{|c|}{$V_{\mathrm{TO}}^{\mathrm{b}}$} \\
\hline-2.26 & 0.539520 & 0.468912 & -0.027354 & 0.000614 \\
\hline-2.08 & 0.603893 & 0.467406 & -0.027372 & 0.000616 \\
\hline-1.78 & 0.785738 & 0.448451 & -0.026057 & 0.000585 \\
\hline-1.56 & 0.941462 & 0.428725 & -0.024491 & 0.000544 \\
\hline-1.25 & 1.324353 & 0.354857 & -0.018317 & 0.000382 \\
\hline-1.07 & 1.428730 & 0.346346 & -0.017664 & 0.000366 \\
\hline-0.77 & 1.515883 & 0.353536 & -0.018211 & 0.000382 \\
\hline-0.54 & 1.657861 & 0.338788 & -0.016849 & 0.000346 \\
\hline \multicolumn{5}{|c|}{$V_{\mathrm{TO}}^{\mathrm{f}}$} \\
\hline-2.26 & 1.058614 & 0.571462 & -0.026232 & 0.000411 \\
\hline-2.08 & 1.058448 & 0.603465 & -0.030062 & 0.000525 \\
\hline-1.78 & 1.016492 & 0.686102 & -0.039767 & 0.000821 \\
\hline-1.56 & 0.989632 & 0.755390 & -0.048332 & 0.001092 \\
\hline-1.25 & 1.391756 & 0.737361 & -0.051244 & 0.001246 \\
\hline-1.07 & 1.928974 & 0.632944 & -0.044782 & 0.001121 \\
\hline-0.77 & 3.212045 & 0.311654 & -0.018641 & 0.000434 \\
\hline-0.54 & 3.506210 & 0.238451 & -0.012321 & 0.000258 \\
\hline \multicolumn{5}{|c|}{$V_{\mathrm{ZAHB}}$} \\
\hline-2.26 & 0.540172 & -0.039658 & 0.002869 & -0.000080 \\
\hline-2.08 & 0.532918 & -0.019872 & 0.001615 & -0.000046 \\
\hline-1.78 & 0.526569 & 0.002118 & 0.000071 & -0.000007 \\
\hline-1.56 & 0.532144 & 0.007506 & -0.000312 & 0.000003 \\
\hline-1.25 & 0.586536 & 0.010611 & -0.000563 & 0.000009 \\
\hline-1.07 & 0.633460 & 0.010605 & -0.000585 & 0.000010 \\
\hline-0.77 & 0.718433 & 0.008860 & -0.000476 & 0.000007 \\
\hline-0.54 & 0.776018 & 0.008516 & -0.000483 & 0.000008 \\
\hline \multicolumn{5}{|c|}{$V_{\text {Bump }}$} \\
\hline-2.26 & -1.642720 & 0.178577 & -0.008972 & 0.000172 \\
\hline-2.08 & -1.650644 & 0.214845 & -0.012553 & 0.000283 \\
\hline-1.78 & -1.349552 & 0.186709 & -0.009940 & 0.000213 \\
\hline-1.56 & -1.232649 & 0.204869 & -0.011501 & 0.000252 \\
\hline-1.25 & -0.825191 & 0.163242 & -0.007843 & 0.000159 \\
\hline-1.07 & -0.614190 & 0.161057 & -0.008018 & 0.000167 \\
\hline-0.77 & -0.324075 & 0.157983 & -0.007189 & 0.000135 \\
\hline-0.54 & 0.149489 & 0.098844 & -0.002696 & 0.000030 \\
\hline \multicolumn{5}{|c|}{$V_{\text {tip }}$} \\
\hline-2.26 & -3.115167 & 0.011530 & -0.000371 & 0.000003 \\
\hline-2.08 & -3.144452 & 0.019023 & -0.000891 & 0.000016 \\
\hline-1.78 & -3.153072 & 0.028388 & -0.001289 & 0.000021 \\
\hline-1.56 & -3.134376 & 0.039542 & -0.001889 & 0.000033 \\
\hline-1.25 & -3.065595 & 0.057298 & -0.002933 & 0.000057 \\
\hline-1.07 & -2.983806 & 0.065208 & -0.003290 & 0.000065 \\
\hline-0.77 & -2.835034 & 0.099049 & -0.004695 & 0.000090 \\
\hline-0.54 & -2.477340 & 0.118030 & -0.005648 & 0.000109 \\
\hline
\end{tabular}

Table 9. As Table 8. Part II: Colours.

\begin{tabular}{ccccc}
\hline \hline$[\mathrm{Fe} / \mathrm{H}]$ & $a_{0}$ & $a_{1}$ & $a_{2}$ & $a_{3}$ \\
\hline \multicolumn{5}{c}{$(B-V)_{\mathrm{TO}}$} \\
\hline-2.26 & -0.191148 & 0.103417 & -0.006435 & 0.000145 \\
-2.08 & -0.168337 & 0.101817 & -0.006405 & 0.000146 \\
-1.78 & -0.111845 & 0.097322 & -0.006293 & 0.000147 \\
-1.56 & -0.059863 & 0.092706 & -0.006109 & 0.000147 \\
-1.25 & 0.052195 & 0.076287 & -0.004949 & 0.000120 \\
-1.07 & 0.133400 & 0.062945 & -0.003886 & 0.000094 \\
-0.77 & 0.269861 & 0.040226 & -0.001963 & 0.000043 \\
-0.54 & 0.329375 & 0.036700 & -0.001614 & 0.000034 \\
\hline \multicolumn{5}{c}{$(B-V)_{\mathrm{RGB}}$} \\
\hline-2.26 & 0.753462 & -0.019023 & 0.001069 & -0.000021 \\
-2.08 & 0.771068 & -0.019883 & 0.001132 & -0.000023 \\
-1.78 & 0.809573 & -0.023631 & 0.001472 & -0.000031 \\
-1.56 & 0.841130 & -0.025759 & 0.001680 & -0.000037 \\
-1.25 & 0.899441 & -0.030508 & 0.002189 & -0.000052 \\
-1.07 & 0.974050 & -0.041904 & 0.003286 & -0.000084 \\
-0.77 & 0.977762 & -0.021655 & 0.001561 & -0.000037 \\
-0.54 & 1.016195 & -0.011496 & 0.000721 & -0.000015 \\
\hline \multicolumn{5}{c}{$(B-V)_{\text {Bump }}$} \\
\hline-2.26 & 0.799812 & -0.001122 & 0.000000 & 0.000002 \\
-2.08 & 0.816658 & -0.002806 & 0.000184 & -0.000004 \\
-1.78 & 0.832050 & -0.000746 & 0.000032 & -0.000001 \\
-1.56 & 0.856822 & -0.001531 & 0.000096 & -0.000001 \\
-1.25 & 0.881010 & 0.003478 & -0.000307 & 0.000009 \\
-1.07 & 0.921353 & 0.000461 & 0.000038 & -0.000002 \\
-0.77 & 0.979223 & 0.003045 & -0.000231 & 0.000007 \\
-0.54 & 1.026973 & 0.005509 & -0.000360 & 0.000108 \\
\hline \multicolumn{5}{c}{00}
\end{tabular}

